\title{
Risking Other People's Money: Gambling, Limited Liability, and Optimal Incentives
}

\author{
Peter M. DeMarzo, Dmitry Livdan, and Alexei Tchistyi*
}

PRELIMINARY AND INCOMPLETE

\begin{abstract}
We consider optimal incentive contracts when managers can, in addition to shirking or diverting funds, increase short term profits by putting the firm at risk of a low probability "disaster." To avoid such risk-taking, investors must cede additional rents to the manager. In a dynamic context, however, because managerial rents must be reduced following poor performance to prevent shirking, poorly performing managers will take on disaster risk even under an optimal contract. This risk taking can be mitigated if disaster states can be identified ex-post by paying the manager a large bonus if the firm survives. But even in this case, if performance is sufficiently weak the manager will forfeit eligibility for a bonus, and again take on disaster risk. When effort costs are convex, reductions in effort incentives is used to limit risk-taking, with a jump to high powered incentives in the gambling region. Our model can explain why suboptimal risk-taking can emerge even when investors are fully rational and managers are compensated optimally.
\end{abstract}

*Peter M. DeMarzo is from Graduate School of Business, Stanford University, email: DeMarzo_Peter@gsb.stanford.edu.

Dmitry Livdan is from Haas School of Business, University of California, Berkeley, email: livdan@haas.berkeley.edu. Alexei Tchistyi is from Haas School of Business, University of California, Berkeley, email: tchistyi@haas.berkeley.edu. 


\section{Introduction}

Investors who entrust their funds to financial institutions such as investment banks, pension and hedge funds typically have little knowledge and/or understanding about how those institutions operate. In particular, it is extremely hard for investors to observe true realized cash flows of the financial institutions as well as correctly evaluate their risk exposure. This asymmetric information creates an opportunity for managers of the financial institutions to enrich themselves at the expense of the investors, which is aided by their ability to swiftly alter risk profiles of the assets under management. To protect their interest, the investors can try to write a contract with a manager of a financial institution that would align manager's objectives with theirs. The goal of this paper is to consider optimal incentive contracts in a setting in which a manager with limited liability privately chooses the riskiness of the project and can privately divert cash flows for her own consumption.

In particular, we consider a stylized model with two-dimensional moral hazard problem. To gain some basic economic insights we start with a one-period version of the model which we later extend to the infinite horizon using continuous time contracting framework. A risk neutral agent (manager) with limited liability runs a project which cash flows depend on its riskiness. The agent can choose between a low-risk and high-risk projects. Compared to the low-risk project, the high-risk project increases the probability of a high cash flow realization, but it also results in high losses in a bad state of nature, which we call "disaster." The possibility of losses in the "disaster" state is eliminated when the low-risk project is chosen. We assume that the lowrisk project is the first best. In addition to risk-taking, the agent can also manipulate the cash flows by diverting cash flows for her consumption. Neither the riskiness of the project nor the cash flow realizations are observed by the investors either ex-ante or ex-post unless the "disaster" state occurs.

The analysis of the one-period model generates a number of key insights. First, we find that although it is possible to write a contract that provides the agents with incentives to choose the low-risk project and not to steal the cash flows, it may be too expensive for the investors to break even. This is the case when the contract terms depend only on cash flows reported by the agent and when the probability of the "disaster" state is low. The economic intuition behind this result is pretty transparent. Any incentive compatible contract must reward the agent for reporting the high cash flow, otherwise the agent would steal it. However, conditioning agent's 
reward on the reported cash flow also creates an incentive for the agent to take the high-risk project. The reason for that is that the agent benefits from the high cash flow and is protected from the losses by the limited liability in the "disaster" state. The contract that induces the agent to choose the low-risk project without stealing cash flows requires very high payoffs for the agent for the non-disaster outcomes. It is so because the agent with limited liability would otherwise ignore the disaster state, which occurs with a low probability, in her calculations of her expected payoff.

Our second finding is that the contract can be much less expensive for the investor to implement if the agent's payoff can be made conditional directly on the disaster state. In the optimal contract the agent receives a big bonus if the project does not generate a big loss in the “disaster" state. This is a cheaper way to provide incentives for the agent to choose the low-risk project, because the high levels of compensation in non-disaster states, which are much more likely to occur than the disaster state, are no longer required. We suggest that in practice this contract can be implemented by giving the manager out-of-money put options on the companies that are likely to be ruined in the disaster state, with a caveat that the manager can collect the payoff from the options only if her company remains in a good shape.

In our continuous time setting, the agent can increase the drift of the cash flow which is driven by Brownian motion, while creating a possibility of disaster, which we model as a Poisson process. Risk-taking occurs when the agent's continuation payoff is below a threshold, which is inversely proportional to the intensity of disaster arrival. Because managerial rents must be reduced following poor performance to prevent fund diversion, poorly performing agents will take on disaster risk even under an optimal contract. Unlike the static model, risk-taking can still happen even when disaster states are contractible. If performance is sufficiently weak the agent will forfeit eligibility for a bonus, and again take on disaster risk.

When effort costs are convex, it is optimal to implement low powered incentives in the no gambling region, while high powered incentives are optimal in the gambling region. Intuitively, stronger incentives not only encourage higher effort, but also make gambling more attractive. As a consequence, in the no gambling region, i.e., with high continuation payoff for the manager, the optimal effort is below not only the first best, but also the second best level in a setting without gambling. On the other hand, in the gambling region, the optimal effort becomes 
substantially higher and can be above the first best level. The high effort, while costly, moves the manager's continuation payoff toward the no gambling region more quickly.

Compared to the setting with the one-dimensional moral hazard problem of DeMarzo and Sannikov (2006), the optimal contract has increased reliance on deferred compensation and provision of financial slack. Another difference from DeMarzo and Sannikov (2006) is that public randomization can sometimes improve the contract. When the agent's continuation payoff drops to the threshold, below which the agent starts gambling, a small reductions in performance could lead to "harsh" punishments. On the other hand, a small improvement in performance in the gambling region could lead to a big increase in the agent's continuation payoff, so that the agent stops gambling immediately.

Our paper bridges two strands of literature: the literature on moral hazard and/or hidden action and the literature on risk-taking. The vast literature on moral hazard (see Salanie (1997) for a survey of static models) has focused mostly on the problem of a principal who wants to induce an agent to exert the "optimal" effort. We build on a hidden action setting similar to the one used by DeMarzo and Sannikov (2006) in continuous time and DeMarzo and Fishman $(2007 \mathrm{a}, \mathrm{b})$ in discrete time.

The risk-shifting was first introduced by Jensen and Meckling (1976) as an agency conflict between equity and debt holders of a levered firm. The agency problem is that the equity would gain all the upside of increasing the risk of the firm's assets-in-place, whereas debt would be responsible for its downside. Recognizing the importance of this agency problem, a large literature studies the impact of non-concave portions (such as puts and calls) of common executive compensations schemes on the risk-shifting incentives of managers who have access to dynamically complete markets. Contributions include Carpenter (2000), Ross (2004), and Basak, Pavlova, and Shapiro (2007). This literature treats the optimal contract as being exogenous.

The most closely related papers to the static version of our model are Diamond (1998), Palomino and Prat (2003), Hellwig (1994), and Biais and Casamatta (1999). Like us, they study a hidden-action moral hazard problem in which the agent controls both effort and the distribution of the outcome. Diamond (1998) and Palomino and Prat (2003) use delegated portfolio management as the economic motivation. Diamond (1989) asks whether, as the cost of effort shrinks relative to the payoffs, the optimal contract converges to the linear contract. He shows that if the agent has several ways to manipulate the outcome, the principal should offer the 
simplest possible compensation scheme, that is, the linear contract. While Diamond (1998) considers only three possible outcomes, Palomino and Prat (2003) allow for a continuum of outcomes. Similar to our setting, the agent in their model has limited liability and can sabotage (misreport) the realized return. They show that the optimal contract is simply a bonus contract the agent is paid a fixed sum if the portfolio return is above a threshold. Also, by using an explicit parameterization of risk, they are able to analyze the sign of inefficiencies in risk taking. Hellwig (1994) and Biais and Casamatta (1999) are interested in the optimal financing of investment projects when managers must exert unobservable effort and can also switch to less profitable riskier ventures. Both papers find that under some technical conditions optimal financial contracts can be implemented by a combination of debt and equity.

The most closely related papers to the dynamic version of our model are by Ou-Yang (2003) and Cadenillas, Cvitanic, and Zapatero (2007). Both papers are executed in a continuous time setting where agent controls both the drift (effort choice) and the volatility (project selection) of the underlying payoff process. Unlike us, neither paper allows for the limited liability of the agent. In addition, we allow for the endogenous liquidation before the terminal date of the project. This assumption is critical since it facilitates "all-or-nothing" strategy from the agent. Implementing this assumption in the economic setting of these two papers is technically infeasible since it would superimpose an optimal stopping problem over their current optimization routine. Finally, Makarov and Plantin (2010) develop a dynamic model of active portfolio management in which fund managers may secretly gamble in order to manipulate their reputation and attract more funds. They solve for the optimal contracts that deter this behavior and show that if investors are short-lived, then the manager must leave rents to investors in order to credibly commit not to gamble. If investors are long-lived, any contract that increases but defers expected bonuses after an outstanding performance is optimal. Contrary to our paper, Makarov and Plantin (2010) consider only observable actions by the agent.

The rest of the paper is organized as follows. The one-period model is presented in Section 2. The continuous time model is discussed in Section 3. The continuous time model with convex costs of effort is analyzed in Section 4 . Section 5 concludes. 


\section{One Period Model}

\subsection{Formulation}

There are two risk-neutral players. First, the principal (investor(s)), owns the company which has value of its assets-in-place equal to A. The principal can add a new project to the company in which case he has to hire the second player, the agent, to run it. The cash flows from the project, $Y(q)$, depend on the degree of its riskiness, $q \in\{0,1\}$, which is controlled by the agent. The project has three possible cash-flow realizations

$$
Y(q)=\left\{\begin{array}{l}
1, \text { with probability } \mu+q \rho \\
0, \text { with probability } 1-\mu-q(\rho+\delta) . \\
-\mathrm{D}, \text { with probability } q \delta
\end{array}\right.
$$

The choice of $q=0$ corresponds to the "safe" project, in which case only cash flows 0 and 1 are possible and its expected value is equal to $\mu \in(0,1)$. The choice of $q=1$ corresponds to the "risky" project. In this case the probability of the highest cash flow, 1 , is increased by $\rho>0$, but a new negative cash flow $-D$ can be realized with probability $\delta>0$. We assume that the "safe" project has higher expected cash flows than the "risky" project

$$
\Delta \equiv \rho-\delta D<0 \text {. }
$$

$D$ can be interpreted as the direct loss from the operations and it could be quite large, but not greater than the value of assets-in-place, i.e. $D<A$. Under this assumption the principal has limited liability with respect to the company, but not the new project.

The agent can take two actions both unobservable by the principle. First, the agent upon privately observing that the realized cash flow is equal to 1 can report to the principal that the cash flow is 0 . By doing so the agent receives a private benefit of $\lambda \in[0,1]$. We interpret diversion of the firm's cash flows as stealing. We assume that the agent can secretly transfer money from the firm's account to his own account. However, other hidden activities that benefit the agent at the expense of the principal may fit the setting of the model as well. For instance, the agent can inefficiently use the firm's cash flows in order to receive non-pecuniary benefits. The fraction $1-\lambda$ represents the cost of diversion, which can be attributed to different kinds of expenses and inefficiencies associated with the diversion. 
Such hidden action setting allows for one-to-one mapping into the hidden effort setting. In this setting the agent chooses a binary effort $e \in\{0,1\}$ at a cost of $\lambda e$. Here $e=1$ stands for "high" (optimal) effort, while $e=0$ stands for "low" (inferior) effort. The payoffs conditional on the joint choice of effort and risk are given by

$$
Y(q, e)=\left\{\begin{array}{l}
1, \text { with probability } \quad e(\mu+q \rho) \\
0, \text { with probability } 1-e(\mu+q \rho)-q \delta \\
-\mathrm{D}, \text { with probability } q \delta
\end{array}\right.
$$

Under the hidden effort interpretation the incentive compatibility constraint enforces the optimal effort.

Privately choosing the "riskiness" of the project, $q$, is the second action the agent can take. We also assume that the agent has limited liability. We interpret limited liability as a disallowance of positive transfers from the agent to the principle, i.e. the agent cannot be legally forced to pay back the principal.

The principal does not observe neither the realized cash flow, $Y$, nor the riskiness, $q$, implemented by the agent and therefore he must rely wholly on the agent to report the cash flow realizations, $\hat{Y}$. We assume that the monitoring is prohibitively costly. Under these assumptions neither $Y$ nor $q$ is contractable.

If the principal agrees to initiate the project, at the time of initiation the principal and the agent sign a contract that governs their relationship over the life of the project. The contract obligates the agent to report realizations of the cash flows to the principal. Without loss of generality, we assume that the contract requires the agent to pay the reported cash flows to the principal immediately. Since there are only three possible cash flows, any contract can be described by three possible payment from the principal to the agent, $w(0), w(1)$, and $w(-D)$ corresponding to reported cash flow 0,1 and $-D$. We assume that the agent has limited liability, so $w(0), w(1)$, and $w(-D)$ must be non-negative.

Next we solve for (i) an optimal contract implementing the safe project; (ii) an optimal contract implementing the risky project; and (iii) an optimal contract when contracting on the disaster state is possible.

\subsection{Optimal contract implementing the safe project}


Here we derive optimal incentive compatible contract that enforces the safe project choice. A contract is optimal if it maximizes the principal's expected payoff, $p(w, q)$, subject to a certain payoff $w_{0}$ for the agent.

The principal's problem is to choose a contract $\{w(0), w(1), w(-D)\}$ that maximizes his expected payoff:

$$
p\left(w_{0}, 0\right)=\max _{w(\cdot) \geq 0} \mathrm{E}[Y-w(Y) \mid q=0]
$$

subject to the promise-keeping (PK thereafter) constraint

$$
(\mathrm{PK}): w_{0}=\mathrm{E}[w(Y) \mid q=0]
$$

the incentive compatibility (IC thereafter) constraint

$$
\text { (IC) }: w(1) \geq w(0)+\lambda,
$$

and the low-risk-taking (LRT thereafter) constraint

$$
(\mathrm{LRT}): \mathrm{E}[w(Y) \mid q=0] \geq \mathrm{E}[w(Y) \mid q=1] .
$$

It is optimal to apply the harshest possible punishment for the disaster outcome, i.e., $w(-D)=0$. Then, LRT constraint becomes equivalent to

$$
\rho(w(1)-w(0)) \leq \delta w(0)
$$

The function $p\left(w_{0}, 0\right)$ represents the highest possible payoff attainable by the principal, given any arbitrary payoff $w_{0}$ to the agent when the safe project is implemented. The PK constraint implies that the agent's expected payoff is $w_{0}$. The IC constraint ensures that when the cash flow of 1 is realized the agent truthfully reports it. The LRT constraint guarantees that the agent selects safe project over the risky project. It is optimal to apply the harshest possible punishment for the disaster outcome, i.e., $w(-D)=0$.

The contracting problem can be written as

$$
\begin{gathered}
p\left(w_{0}, 0\right)=\max _{w(\cdot) \geq 0} \mu(1-w(1))+(1-\mu)(-w(0)), \\
\text { s.t. }(\mathrm{PK}): w_{0}=\mu w(1)+(1-\mu) w(0), \\
(\mathrm{IC}): w(1) \geq w(0)+\lambda, \\
(\mathrm{LRT}): \rho(w(1)-w(0)) \leq \delta w(0),
\end{gathered}
$$

Combining (11) and (12) we obtain a low bound on $w(0)$ : 


$$
w(0) \geq \frac{\lambda \rho}{\delta}
$$

The payoff for the high cash flow $w(1)$ must be at least $\lambda$ more. Thus, the lowest expected payoff for the agent under a contract that satisfies both IC and LRT constraints is given by

$$
w^{s} \equiv \frac{\lambda \rho}{\delta}+\mu \lambda
$$

Finally, we can substitute the PK constraint (10) into the objective function (9) to obtain

$$
p\left(w_{0}, 0\right)=p^{0}-w_{0}, \text { for } w_{0} \geq w^{s} .
$$

We summarize our findings in Proposition 1.

PROPOSITION 1: The optimal incentive compatible contract implementing safe project is $\left\{w(0)=w_{0}-\mu \lambda, w(1)=w_{0}+(1-\mu) \lambda, w(-D)=0\right\} \forall w_{0} \geq w^{s}$. The principal's expected payoff is given by

$$
p\left(w_{0}, 0\right)=p^{0}-w_{0}, \text { for } w_{0} \geq w^{s},
$$

where the minimum expected payoff to the agent is equal to

$$
w^{s}=\frac{\lambda \rho}{\delta}+\mu \lambda
$$

Since $\delta$ can be arbitrarily small (but not necessarily the expected losses since $D$ can be made arbitrarily large), the optimal contract can become too expensive for the principal to implement. This happens since in order to jointly satisfy IC and LRT constraints the agent has to be given high payoffs when cash flows are either 0 or 1 .

Intuitively, in order to provide the agent with incentives to reveal cash flows truthfully the agent should be rewarded for reporting the high cash flow, as specified by the IC constraint. This, however, creates incentives for gambling. The LRT constraint says that the expected benefit of gambling for the agent $\rho(w(1)-w(0))$ should be less or equal than the expected loss $\delta w(0)$. Since the punishment of the agent for creating disaster is bounded due to limited liability, in order to prevent gambling the agent should be given an extra rent $w(0)$ for delivering zero cash flow. Since the loss from gambling $\delta w(0)$ is proportional to $\delta$, the rent $w(0)$ must be increased when $\delta$ declines. As a result, gambling is more costly to prevent when the probability of disaster is low. 


\subsection{Optimal contract implementing the risky project}

We now derive an optimal contract that implements the risky project. The contracting problem is to find a contract $\{w(0), w(1), w(-D)\}$ that maximizes the principal's expected payoff:

$$
p\left(w_{0} ; 1\right)=\max _{w(\cdot) \geq 0} \mathrm{E}[Y-w(Y) \mid q=1],
$$

subject to the PK and IC constraints

$$
(\mathrm{PK}): w_{0}=\mathrm{E}[w(Y) \mid q=1]
$$

and

$$
\text { (IC) }: w(1) \geq w(0)+\lambda \text {. }
$$

Combining (16) and (17) gives

$$
p\left(w_{0}, 1\right)=\mu+\rho-\delta D-w_{0},
$$

i.e., the principal's payoff is equal to the expected cash flow minus the expected payoff to the agent. Since there is no LRT constraint, both $w(-D)$ and $w(0)$ can be as low as zero, while $w(1)$ should be at least $\lambda$. As the result, the lowest possible expected payoff for the agent when gambling is allowed is equal to

$$
w^{g} \equiv(\mu+\rho) \lambda
$$

Thus, we have Proposition 2.

PROPOSITION 2: The optimal contract implementing the risky project is given by $w(0)=\frac{w_{0}-(\mu+\rho) \lambda}{1-\delta}, w(1)=\frac{w_{0}+(1-\mu-\rho-\delta) \lambda}{1-\delta}, w(-D)=0$ for $w_{0} \geq(\mu+\rho) \lambda$.

The

principal's payoff is given by

$$
p\left(w_{0}, 1\right)=\mu+\rho-\delta D-w_{0}, \text { for } w_{0} \geq w^{g},
$$

where the minimum expected payoff to the agent is equal to

$$
w^{g}=(\mu+\rho) \lambda \text {. }
$$

Corollaries 1 and 2 highlight our next set of results. 
COROLLARY 1: The lowest agent's compensation under an optimal contract implementing risky project is smaller than the lowest compensation under an optimal contract implementing safe project, i.e., $w^{g}<w^{s}$.

This result follows immediately from the fact that $\delta<1$. Thus, the contract allowing the risky project is less expensive than the one that implements the safe project in terms of the agent's compensation. At the same time, gambling reduces the expected cash flow by $\delta D-\rho$. Thus, the principal would choose the safe project only if the expected losses from gambling outweigh the extra cost of the agent's compensation.

COROLLARY 2: When the principal can choose the lowest level of the agent's compensations, the principle is better off implementing the safe project if

$$
\delta D-\rho \geq \lambda \rho \frac{1-\delta}{\delta} .
$$

We note that the right hand side of the inequality (21) goes to infinity when $\delta$ goes to zero. Thus, when the probability of the disaster $\delta$ is low and $D$ is large so that the safe project is strictly better than the risky one, the principal would prefer the risky project or would undertake no project.

\subsection{Optimal Contract Conditional on the Disaster State}

In this subsection, we consider a setting with an observable disaster state, e.g., an earthquake or a major financial crisis. Thus, it is now possible to write a contract conditional not only on reported cash flows, but also on states of nature.

We modify our previous setting as follows. At time zero nature makes a draw: with probability $\delta$ it draws a "disaster" state, and it draws "good" state with probability $1-\delta$. Ex ante, neither principal nor agent knows the exact state of nature, but ex post the true state of nature is a public knowledge.

In the "disaster" state, the cash flows conditional on the risk $q \in\{0,1\}$, are as follows 


$$
Y_{d}(q)=\left\{\begin{array}{l}
1, \text { with probability } 0, \\
0, \text { with probability } 1-q, \\
-D, \text { with probability } q .
\end{array}\right.
$$

while in the "good" state the cash flows are

$$
Y_{g}(q)=\left\{\begin{array}{l}
1, \text { with probability }(\mu+q \rho) /(1-\delta), \\
0, \text { with probability } 1-(\mu+q \rho) /(1-\delta), \\
-D, \text { with probability } 0 .
\end{array}\right.
$$

The cash flows (22) and (23), which are conditional on the states of nature $n \in\{g, d\}$, are fully consistent with our previous setup, since the unconditional probabilities of the cash flows are the same as in (1).

We now derive an optimal contract that implements the safe project. The crucial difference from the previous setup is that the transfer to the agent, $w(\hat{y}, n)$, is not only a function of the reported cash flow, $\hat{y}$, but also depends on the state of nature $n$.

The principal's problem is to choose a contract $\{w(0, g), w(1, g), w(0, d), w(-D, d)\}$ that maximizes his expected payoff:

$$
p\left(w_{0}, 0\right)=\max _{w(\cdot) \geq 0} \mathrm{E}[Y-w(Y, n) \mid q=0],
$$

subject to the promise-keeping constraint

$$
(\mathrm{PK}): w_{0}=\mathrm{E}[w(Y, n) \mid q=0]
$$

the incentive compatibility constraint

$$
\text { (IC) }: w(1, n) \geq w(0, n)+\lambda,
$$

and the low-risk-taking constraint

$$
(\mathrm{LRT}): \mathrm{E}[w(Y, n) \mid q=0] \geq \mathrm{E}[w(Y, n) \mid q=1] .
$$

Using (22) and (23) and the fact that it is optimal to have $w(-D, d)=0$, the constraints can be written as

$$
\begin{gathered}
(\mathrm{PK}): w_{0}=\mu w(1, g)+(1-\mu-\delta) w(0, g)+\delta w(0, d), \\
(\mathrm{IC}): w(1, g)-w(0, g) \geq \lambda, \\
(\mathrm{LRT}): \rho(w(1, g)-w(0, g)) \leq \delta w(0, d),
\end{gathered}
$$

The lowest possible payments to the agent that satisfy both (IC) and (LRT) constraints are $w(1, g)=\lambda, w(0, g)=0, w(0, d)=\rho \lambda / \delta$. Substituting them into (PK) gives that the lowest 
expected payment for the agent is equal to $\lambda(\mu+\rho)=w^{g}$, and the corresponding payoff for the principal $\mu-w^{g}$.

Thus, the principal can implement the safe project writing a contract conditional on the disaster state while giving the agent the same minimum level of compensation required to implement the risky project. This is achieved by giving a bonus $b=\rho \lambda / \delta$ for zero cash flow in the disaster state. Proposition 3 summarizes our results.

PROPOSITION 3: An optimal contract conditional on the disaster state implements the safe project and is characterized by payoffs $w(0)=w_{0}-\delta b-\mu \lambda, w(1)=w_{0}-\delta b+(1-\mu) \lambda, w(-D)=0$ to the agent conditional on cash flows $0,1,-D$, and additional bonus $b=\rho \lambda / \delta$ given only in the disaster state for the zero cash flow. The principal's expected payoff is given by

$$
p\left(w_{0}, 0\right)=p^{0}-w_{0}, \text { for } w_{0} \geq w^{g},
$$

where the minimum expected payoff to the agent $\bar{a}$ is equal to

$$
w^{g}=(\mu+\rho) \lambda .
$$

The intuition behind this result is transparent and as follows. Depending on the state of nature, cash flow 0 can be either bad or good outcome -- it is bad in the "good" state and good in the "disaster" state. When contracting on the states of nature is not allowed as in Propositions 1, the agent has to be given high rewards for both 0 and 1 cash flows in order to implement the safe project, which makes the optimal contract expensive. When contracting on the states of nature is

allowed, the contract can differentiate between cash flows 0 and 1 , and, therefore, it is not necessary to promise the agent high payoffs for either cash flow in the good state of nature. Instead, it is optimal to give the agent bonus $b$ for cash flow 0 in the disaster state of nature. While this bonus becomes high when $\delta$ is small, the expected bonus, $\delta b=\mu+\rho$, remains small. As a result, the cost of managerial compensation remains small.

\subsection{Implementation}

In practice, conditioning on states of nature while possible is quite challenging. It is so because states of nature are difficult to categorize and verify. However, our optimal contract requires 
conditioning only on the "extreme" states of nature, such as a major financial crisis. During such a crisis a number of major financial institutions either go bankrupt or their equity suffers extreme losses. Therefore, one practical way to implement our contract is as follows. At the time when the whole economy is doing well, managers who have either authority or ability to change the riskiness of their projects should be awarded out-of-money put options on other companies in the same line of business which are likely to be ruined in the case of disaster. This implementation is relatively inexpensive at the award time since these options would be cheap. However they would result in large payoffs to their holders in the case of the disaster. The necessary caveat is that the manager would get this payoff only if his company stays afloat (payoff 0 in the “disaster" state in our model). Manager would rationally anticipate this large reward at the outset and would implement the safe project.

These contracts are, however, not observed in practice. One argument against compensation like that is that it provides managers with extra incentives to take down their competitors. Our paper is the first to point out the particular benefits of using put options on other companies as a part of managerial compensations. In light of the recent financial crisis, the benefits of such compensation can outweigh its downside, since losses from excessive risktaking it helps to prevent can be quite large. A comprehensive cost-benefit analysis of such executive compensation should be executed in a general equilibrium setting which is beyond the scope of this paper and is left for future research.

\section{Continuous Time Model}

\subsection{Formulation}

This section extends the one-period model to the continuous time, infinite horizon setting. The agent manages a project that generates a continuous cash flow stream, $Y_{t}$, that depends on the riskiness of the project, $q_{t} \in$ $\{0,1\}$, as follows

$$
d Y_{t}=\left(\mu+\rho q_{t}\right) d t+\sigma d Z_{t}-D q_{t} d N_{t}
$$

where $Z_{t}$ is a standard Brownian motion, and $\sigma$ is the volatility of cash flow process. The drift of the cash flow process is $\mu$ in the "low" risk regime, $q_{t}=0$, and is increased by $\rho>0$ in the "high" risk regime, $q_{t}=1$. In addition, the high risk regime exposes the project to a possibility of a "disaster" outcome, in which case the project generates a one-time large loss $D \geq 0$ and gets liquidated immediately. "Disaster" process $N$ is a 
standard Poisson process with intensity $\delta$ that governs arrivals of disaster state when $d N_{t}=1$. A disaster state results in a "disaster outcome" for the project, i.e., loss of $D$, only if the project was in the high risk regime at the moment when the disaster arrived.

We consider a two dimensional agency problem stemming from hidden actions taken by the agent. The first dimension is that the riskiness of project can be privately changed by the agent at any time and is not observable or verifiable by the investors, (except the times when a disaster takes place). We assume that $\rho<\delta D$, i.e., switching to the high risk regime leads to lower cash flows in expectation.

The second dimension is that the agent observes the realized cash flows $Y$, but the principal does not. The agent reports cash flows $\left\{\hat{Y}_{t} ; t \geq 0\right\}$ to the principal, where the difference between $Y$ and $\hat{Y}$ is determined by the agent's hidden actions. The principal receives only the reported cash flows $d \hat{Y}_{t}$ from the agent.

The contract specifies compensation for the agent $d I_{t}$, as well as a termination times $\tau_{L}$ and $\tau_{D}$, that are based on the agent's reports and disaster realizations. We begin our analysis assuming that the contract cannot be made conditional on the occurrence of the disaster, unless the disaster outcome occurs, in which case the project is terminated at time

$$
\tau_{D} \equiv \inf \left\{t: d N_{t}=1 \& q_{t}=1\right\} .
$$

Thus, the termination time $\tau_{L}$ and agent's compensation, $d I_{t}$, depend only on the history of the agent's reports.

In this section we model the agency problem by allowing the agent to divert cash flows for his own private benefit. The agent receives a fraction $\lambda \in(0,1]$ of the cash flows he diverts; if $\lambda<1$, there are deadweight costs of concealing and diverting funds. The agent can also exaggerate cash flows by putting his own money back into the project. By altering the cash flow process in this way, the agent receives a total flow of income of

$$
\left[d Y_{t}-d \hat{Y}_{t}\right]^{\lambda}+d I_{t}, \text { where }\left[d Y_{t}-d \hat{Y}_{t}\right]^{\lambda} \equiv \underbrace{\lambda\left(d Y_{t}-d \hat{Y}_{t}\right)^{+}}_{\text {diversion }}-\underbrace{\left(d Y_{t}-d \hat{Y}_{t}\right)^{-}}_{\text {over-reporting }} .
$$

The agent is risk neutral and discounts his consumption at rate $\gamma$. The agent maintains a private savings account, from which he consumes and into which he deposits his income. The principal cannot observe the balance of the agent's savings account. The agent's balance $S_{t}$ grows at interest rate $\rho<\gamma$.

$$
d S_{t}=\rho S_{t} d t+\left[d Y_{t}-d \hat{Y}_{t}\right]^{\lambda}+d I_{t}-d C_{t}
$$


where $d C_{t} \geq 0$ is the agent's consumption at time $t$. The agent must maintain a nonnegative balance on his account, that is, $S_{t} \geq 0$.

Once the contract is terminated, the agent receives payoff of zero. Therefore, the agent's total expected payoff from the contract at date zero is given by

$$
W_{0}=E\left[\int_{0}^{\tau} e^{-\gamma s} d C_{s}\right]
$$

where $\tau \equiv \min \left\{\tau_{L}, \tau_{D}\right\}$.

The principal discounts cash flows at rate $r$, such that $\gamma>r \geq \rho$. Once the contract is terminated, she receives expected liquidation payoff $L \geq 0$ unless the termination was caused by the "disaster" outcome, in which case the liquidation value of the project is $L-D$. The principal's total expected profit at date zero is then

$$
p_{0}=E\left[\int_{0}^{\tau} e^{-r s}\left(d \hat{Y}_{s}-d I_{s}\right)+e^{-r \tau}\left(L 1_{\tau_{L}<\tau_{D}}+(L-D) 1_{\tau_{D} \leq \tau_{L}}\right)\right]
$$

The project requires the start-up external capital of $K \geq 0$. The principal offers to contribute this capital in exchange for a contract $\left\{\tau_{L}, I\right\}$ that specifies a termination time $\tau_{L} \wedge \tau_{D}$ and payments $\left\{I_{t} ; 0 \leq t \leq \tau_{L} \wedge \tau_{D}\right\}$ that are based on reports $\hat{Y}$. Formally, $I$ is a $\hat{Y}$-measurable continuous process, and $\tau_{L}$ is a $\hat{Y}$-measurable stopping time.

In response to a contract $\left\{\tau_{L}, I\right\}$, the agent chooses a feasible strategy to maximize his expected payoff. A feasible strategy is a triple of processes $\{q, C, \hat{Y}\}$ adapted to $Y$ such that

(i) $q \in\{0,1\}$,

(ii) $\quad \hat{Y}$ is continuous and, if $\lambda<1, Y_{t}-\hat{Y}_{t}$ has bounded variation, ${ }^{10}$

(iii) $\quad C_{t}$ is non-decreasing, and

(iv) the savings process, defined by (2), stays nonnegative.

The agent's strategy $\{q, C, \hat{Y}\}$ is incentive compatible if it maximizes his total expected payoff $W_{0}$ given a contract $\left(\tau_{L}, I\right)$. An incentive compatible contract refers to a quintuple $\left\{\tau_{L}, I, q, C, \hat{Y}\right\}$ that includes the agent's recommended strategies.

The optimal contracting problem is to find an incentive compatible contract $\left\{\tau_{L}, I, q, C, \hat{Y}\right\}$ that maximizes the principal's profit subject to delivering the agent an initial required payoff $W_{0}$. By varying $W_{0}$ we can use this solution to consider different divisions of bargaining power between the agent and the principal. 
For example, if the agent enjoys all the bargaining power due to competition between principals, then the agent must receive the maximal value of $W_{0}$ subject to the constraint that the principal's profit be at least zero.

\subsection{Derivation of the Optimal Contract}

We solve the problem of finding an optimal contract in three steps. First, we show that it is sufficient to look for an optimal contract within a smaller class of contracts, namely, contracts in which the agent chooses to report cash flows truthfully and maintain zero savings. Second, we consider a relaxed problem by ignoring the possibility that the agent can save secretly. Third, we show that the contract is fully incentive compatible even when the agent can save secretly.

We begin with a revelation principle type of result:

LEMMA A: There exists an optimal contract in which the agent (i) chooses to tell the truth, and (ii) maintains zero savings.

The intuition for this result is straightforward - it is inefficient for the agent to conceal and divert cash flows $(\lambda \leq 1)$ or to save them $(\rho \leq r)$, as we could improve the contract by having the principal save and make direct payments to the agent. Thus, we will look for an optimal contract in which truth telling and zero savings are incentive compatible.

\subsubsection{The Optimal Contract with Non-Contractible Disaster Process}

Note that if the agent could not save, then he would not be able to over-report cash flows and he would consume all income as it is received. Thus,

$$
d C_{t}=d I_{t}+\lambda\left(d Y_{t}-d \hat{Y}_{t}\right)
$$

We relax the problem by restricting the agent's savings so that (52) holds and allowing the agent to steal only at a bounded rate. After we find an optimal contract for the relaxed problem, we show that it remains incentive compatible even if the agent can save secretly or steal at an unbounded rate. 
One challenge when working in a dynamic setting is the complexity of the contract space. Here, the contract can depend on the entire path of reported cash flows $\hat{Y}$. This makes it difficult to evaluate the agent's incentives in a tractable way. Thus, our first task is to find a convenient representation of the agent's incentives. Define the agent's promised value $W_{t}(\hat{Y}, q)$ after a history of reports $\left(\hat{Y}_{s}, 0 \leq s \leq t\right)$ to be the total expected payoff the agent receives, from transfers and termination utility, if he tells the truth and chooses risk process $q$ after time $t$ :

$$
W_{t}(\hat{Y}, q)=E_{t}\left[\int_{t}^{\tau} e^{-\gamma(s-t)} d C_{s}\right]
$$

The following result provides a useful representation of $W_{t}(\hat{Y}, q)$.

LEMMA B: At any moment of time $t \leq \tau$, there is a sensitivity $\beta_{t}(\hat{Y}, q)$ of the agent's continuation value towards his report and public randomization process $R_{t}(\hat{Y}, q)$ such that

$$
d W_{t}=\left(\gamma+\delta q_{t}\right) W_{t} d t-d I_{t}+\beta_{t}(\hat{Y}, q)\left(d \hat{Y}_{t}-\left(\mu+\rho q_{t}\right) d t\right)-q_{t} W_{t} d N_{t}+\left(d R_{t}(\hat{Y}, q)-E\left[d R_{t}(\hat{Y}, q)\right]\right) .
$$

This sensitivity $\beta_{t}(\hat{Y}, q)$ is determined by the agent's past reports $\hat{Y}_{s}$, and riskiness of the project $q_{s}, 0 \leq s \leq t$.

Proof of Lemma B: See Appendix.

Informally, the agent has incentives not to steal cash flows if he gets at least $\lambda$ of promised value for each reported dollar, that is, if $\beta_{t} \geq \lambda$. If this condition holds for all $t$ then the agent's payoff will always integrate to less than his promised value if he deviates. If this condition fails on a set of positive measure, the agent can obtain at least a little bit more than his promised value if he underreports cash when $\beta_{t}<\lambda$.

In addition, the agent has incentives to switch to the high-risk regime to increase the project cash flows. However, this can lead to a disaster outcome resulting in the loss of continuation payoff for the agent. Intuitively, the agent would be willing to take on more risk only if his continuation payoff is below a certain threshold. Indeed, if the agent decides to switch to the high-risk regime at time $t$, the cash flow will be increased by $\rho d t$. If the agent truthfully reports the cash flow and switches back to low-risk regime at time $t+d t$, it results in an increase of the agent's continuation payoff by $\beta_{t} \rho d t$. However, with probability $\delta d t$ this could lead to a 
disaster outcome between time $t$ and $t+d t$, and the loss of continuation payoff $W_{t}$. Thus, choosing the high-risk regime is incentive compatible if and only if $\beta_{t} \rho d t>\delta W_{t} d t$, or $W_{t}<\beta_{t} \frac{\rho}{\delta}$.

LEMMA C: If the agent cannot save, truth-telling is incentive compatible if and only if $\beta_{t} \geq \lambda$ for all $t<\tau$. Moreover, it is optimal for the agent to choose the low-risk regime whenever $W_{t} \geq \beta_{t} \frac{\rho}{\delta}$ and switch to the highrisk regime whenever $W_{t}<\beta_{t} \frac{\rho}{\delta}$.

Proof of Lemma C: See Appendix.

Now we use the dynamic programming approach to determine the most profitable way for the principal to deliver the agent any value $W$. Here we present an informal argument, which we formalize in the proof of Proposition 1 in the Appendix. Denote by $p(W)$ the principal's value function (the highest profit to the principal that can be obtained from a contract that provides the agent the payoff $W$ ).

Because the principal has the option to provide the agent with $W$ by paying a lump-sum transfer of $d I>$ 0 and moving to the optimal contract with payoff $W-d I$,

$$
p(W) \geq p(W-d I)-d I .
$$

Equation (55) implies that $b^{\prime}(W) \geq-1$ for all $W$; that is, the marginal cost of compensating the agent can never exceed the cost of an immediate transfer. Define $W^{\mathrm{C}}$ as the lowest value such that $b^{\prime}\left(W^{\mathrm{C}}\right)=-1$. Then it is optimal to pay the agent according to

$$
d I=\max \left(W-W^{C}, 0\right) .
$$

These transfers, and the option to terminate, keep the agent's promised value between 0 and $W^{\mathrm{C}}$. Within this range, Lemmas B and C imply that the agent's promised value evolves according to

$$
d W_{t}=\left(\gamma+\delta q_{t}\right) W_{t} d t+\beta_{t} \sigma d Z_{t}-q_{t} W_{t} d N_{t}+\left(d R_{t}-E\left[d R_{t}\right]\right)
$$

when the agent is telling the truth. We need to determine the sensitivity $\beta$ of the agent's value to reported cash flows and the randomization process $R$ that maximize the principal's value. 
Let $d \Delta_{t}^{R}$ denote the stochastic probability that the randomization process $R$ results in a jump in the agent's continuation payoff between $t$ and $t+d t$. Let $J_{t}^{R}$ denote the size of this jump. Using Ito's lemma, the principal's expected cash flows and changes in contract value are given by

$$
\begin{aligned}
E[d Y+d p(W)]= & \left(\mu+\rho q+(\gamma+\delta q) W b^{\prime}(W)+\frac{1}{2} \beta^{2} \sigma^{2} p^{\prime \prime}(W)+\delta q(L-D-p(W))\right) d t+ \\
& E\left[\beta \sigma p^{\prime}(W) d Z+\left(p\left(W+J^{R}\right)-p(W)\right) d \Delta^{R}-p^{\prime}(W) J^{R} d \Delta^{R}\right] .
\end{aligned}
$$

Because at the optimum the principal should earn an instantaneous total return equal to the discount rate, $r$, we have the following equation for the value function:

$$
\begin{gathered}
r p(W) d t=\max _{\beta, q, J^{R}, \Delta^{R}}\left(\mu+\rho q+(\gamma+\delta q) W p^{\prime}(W)+\frac{1}{2} \beta^{2} \sigma^{2} p^{\prime \prime}(W)+\delta q(L-D-p(W))\right) d t+ \\
E\left[\beta \sigma p^{\prime}(W) d Z+\left(p\left(W+J^{R}\right)-p(W)-p^{\prime}(W) J^{R}\right) d \Delta^{R}\right] \\
\text { s.t. } \beta \geq \lambda \\
q=1 \Leftrightarrow \beta>\frac{\delta W}{\rho} \\
J^{R} \geq-W \\
d \Delta^{R} \geq 0
\end{gathered}
$$

First we note that optimal randomization assures that function $p$ is weakly concave. If $p(W)$ is convex around $W$, then it is optimal to randomize around $W$, since $\left(p\left(W+J^{R}\right)-p(W)-p^{\prime}(W) J^{R}\right)$ is positive for some $J^{R}$. However, the randomization makes $p(W)$ affine in $W$, and hence $p$ "' $(W)=0$. Thus, $p(W)$ cannot be convex, and the last term in equation (59) is zero.

Equation (59) can be rewritten as follows:

$$
r p(W)=\mu+\gamma W p^{\prime}(W)+\max _{\beta, q} \frac{1}{2} \beta^{2} \sigma^{2} p^{\prime \prime}(W)+q \delta\left(\left(\frac{\mu_{1}}{\delta}-D\right)+\left(L-\left(p(W)-W p^{\prime}(W)\right)\right)\right) .
$$

Given the concavity of $p, p^{\prime \prime}(W) \leq 0$ and $L-\left(p(W)-W p^{\prime}(W)\right) \leq 0$. In addition, $\frac{\rho}{\delta}-D<0$, because the high-risk project is dominated by the low risk project. As a result, the smallest possible $\beta$ and $q$ are optimal. Thus, $\beta=\lambda$, and $q=1$ for $W<W^{S} \equiv \lambda \frac{\rho}{\delta}$, and $q=0$ for $W>W^{S}$. Intuitively, since the inefficiency in this model results from early termination and gambling, choosing lowest possible $\beta$ has a double benefit. First, reducing the risk to 
the agent lowers the probability that the agent's promised value falls to zero. Second, lower $\beta$ reduces incentives for the agent to gamble.

We require three boundary conditions to pin down a solution to this equation and the boundary $W^{\mathrm{C}}$. The first boundary condition arises because the principal must terminate the contract to hold the agent's value to 0 , so $p(0)=L$. The second boundary condition is the usual "smooth pasting" condition - the first derivatives must agree at the boundary - and so $p^{\prime}\left(W^{\mathrm{C}}\right)=-1$. The final boundary condition is the "super contact" condition for the optimality of $W^{\mathrm{C}}$, which requires that the second derivatives match at the boundary. The principal's value function therefore satisfies the following second-order ordinary differential equation:

$$
r p(W)=\mu+\gamma W p^{\prime}(W)+\frac{1}{2} \lambda^{2} \sigma^{2} p^{\prime \prime}(W), \quad W^{S} \leq W \leq W^{\mathrm{C}},
$$

with $p(W)=p\left(W^{\mathrm{C}}\right)-\left(W-W^{\mathrm{C}}\right)$ for $W>W^{\mathrm{C}}$. This condition implies that $p^{\prime \prime}\left(W^{\mathrm{C}}\right)=0$, or equivalently, using equation (62),

$$
r p\left(W^{C}\right)+\gamma W^{C}=\mu \text {. }
$$

This boundary condition has a natural interpretation: It is beneficial to postpone payment to the agent by making $W^{\mathrm{C}}$ larger because doing so reduces the risk of early termination. Postponing payment is sensible until the boundary (63), when the principal and agent's required expected returns exhaust the available expected cash flows.

The following proposition formalizes our findings:

PROPOSITION 5: The contract that maximizes the principal's profit and delivers the value $W_{0} \in\left[0, W^{C}\right]$ to the agent takes the following form: $W_{t}$ evolves according to

$$
d W_{t}=\left(\gamma+\delta q_{t}\right) W_{t} d t-d I_{t}+\lambda\left(d \hat{Y}_{t}-\left(\mu+\rho q_{t}\right) d t\right)-q_{t} W_{t} d N_{t}-J\left(d K_{t}^{S}-\Lambda_{t}^{S}\right)+J\left(d K_{t}^{P}-\Lambda_{t}^{P}\right) .
$$

When $W_{t} \in\left[0, W^{C}\right), d I_{t}=0$. When $W_{t}=W^{C}$, payments $d I_{t}$ cause $W_{t}$ to reflect at $W^{C}$. If $W_{0}>W^{C}$, an immediate payment $W_{0}-W^{C}$ is made. The contract is immediately terminated when the disaster outcome occurs. The contract is also terminated when $W_{t}$ reaches 0 . Jumps of the size $J=W^{S}-W^{P}$ can happen when $W_{t}$ reaches thresholds $W^{P}$ or $W^{S}$. The cumulative intensities $\Lambda_{t}^{S}$ and $\Lambda_{t}^{P}$ of the jumps are given by 


$$
\begin{gathered}
d \Lambda_{t}^{S}=-1_{\left\{W_{t}=W^{S}\right\}} \frac{\left[(\gamma+\delta) W_{t} d t+\lambda\left(d \hat{Y}_{t}-(\mu+\rho) d t\right)\right]^{-}}{\mathrm{W}^{\mathrm{S}}-\mathrm{W}^{\mathrm{P}}}, \\
d \Lambda_{t}^{P}=1_{\left\{W_{t}=W^{P}\right\}} \frac{\left[(\gamma+\delta) W_{t} d t+\lambda\left(d \hat{Y}_{t}-(\mu+\rho) d t\right)\right]^{+}}{\mathrm{W}^{\mathrm{S}}-\mathrm{W}^{\mathrm{P}}} .
\end{gathered}
$$

If $\mathrm{W}_{0} \in\left(\mathrm{W}^{P}, W^{S}\right)$, the agent's time zero continuation payoff will be $W^{P}$ with probability $\frac{W^{S}-W_{0}}{W^{S}-W^{P}}$, and $W^{S}$ with probability $\frac{W_{0}-W^{P}}{W^{S}-W^{P}}$, when the project is initiated. It is optimal for the agent to choose the high-risk regime whenever $W_{t}<W^{S}$ and the low-risk regime whenever $W_{t} \geq W^{S}$. The principal's expected payoff at any point is given by a weakly concave continuously differentiable function $p(W)$ that solves

$$
(r+\delta) p(W)=(\mu+\rho-\delta(D-L))+(\gamma+\delta) W p^{\prime}(W)+\frac{1}{2} \lambda^{2} \sigma^{2} p^{\prime \prime}(W),
$$

on the interval $\left[W^{0}, W^{P}\right]$,

$$
r p(W)=\mu+\gamma W p^{\prime}(W)+\frac{1}{2} \lambda^{2} \sigma^{2} p^{\prime \prime}(W),
$$

on the interval $\left[W^{S}, W^{C}\right]$, and is given by

$$
p(W)=p\left(W^{P}\right)+\frac{p\left(W^{S}\right)-p\left(W^{P}\right)}{W^{S}-W^{P}}\left(W-W^{P}\right),
$$

on the interval $\left(W^{P}, W^{S}\right)$.

When

$$
\delta D+r L \geq \mu+\rho,
$$

the agent is never allowed to gambles, i.e., $W^{P}=0$.

When

$$
\delta D+r L<\mu+\rho
$$

and

$$
(r+\delta) p\left(W^{S}\right)-(\gamma+\delta) p^{\prime}\left(W^{S}\right) W^{S} \leq \mu+\rho-\delta(D-L),
$$

then there are no jumps in the agent's continuation payoff, i.e., $W^{P}=W^{S}$.

When $\delta D+r L<\mu+\rho$, and

$$
(r+\delta) p\left(W^{S}\right)-(\gamma+\delta) p^{\prime}\left(W^{S}\right) W^{S}>\mu+\rho-\delta(D-L),
$$

then $0<W^{P}<W^{S}$ and $W^{P}$ solves

$$
(r+\delta) p\left(W^{P}\right)-(\gamma+\delta) p^{\prime}\left(W^{P}\right) W^{P}=\mu+\rho-\delta(D-L) .
$$

The additional boundary conditions are given by 


$$
\begin{aligned}
& p^{\prime}(W)=-1 \text { for } W \geq W^{C}, \\
& r p\left(W^{C}\right)+\gamma W^{C}=\mu, \\
& p(0)=L,
\end{aligned}
$$

Proof of Proposition 5: See Appendix

Condition (70) has a simple and intuitive interpretation. It says that gambling cannot be allowed under any circumstances when the expected cash flows $\mu+\rho$ generated by the project are less than the expected loss $\delta D$ due to gambling and the opportunity cost of delaying liquidation $r L$. When gambling is allowed under the optimal contract, then no randomization is needed when condition (71) is satisfied. This condition guarantees that the principal's value function $p(W)$ is concave, i.e., $p^{\prime \prime}(W) \leq 0$.

When condition (71) is not satisfied, randomization is needed. Intuitively, the value function would be convex on interval $\left(W^{P}, W^{S}\right)$ without jumps. This means that it should be optimal to increase the volatility of $W$ on this interval. In the limit as the volatility goes to infinity, the $W$ jumps between $W^{P}$ and $W^{S}$. The size of the jump is determined by condition (72), which is equivalent to $p^{\prime \prime}\left(W^{P}\right)=0$.

One immediate implication of Proposition 5 is that the optimal contract relies on deferred compensation when gambling is possible more than when the agent cannot increase the riskiness of the project, i.e., $\mu=0$ and $\delta=0$. In both cases the payoffs at the consumption boundary $W^{C}$ and $b\left(W^{C}\right)$ lie on the straight line (74), whose slope is negative. Since the gambling is inefficient, the principal's expected payoff given by the value function $p(W)$ is lower. As a result, the optimal consumption boundary is $W^{C}$ is higher when gambling is possible.

\subsubsection{The Optimal Contract with Contractible Disaster Process}

In this section, we derive the optimal contract when the disaster process is observable and contractible. As before, when the disaster arrives and the risk is high, the disaster outcome occurs, resulting in immediate liquidation of the project and the negative payoff of $L-D$ to the investors. However, unlike the previous setting, the terms of the contract can be conditional on the event when the disaster arrives and the risk is low. We will call continuation payoff adjustments conditional on such an event "bonuses." 
It is straightforward to modify Lemma B for the setting with bonuses.

LEMMA D: At any moment of time $t \leq \tau$, there is a sensitivity $\beta_{t}(\hat{Y}, q)$ of the agent's continuation value towards his report and a bonus process $B_{t}(\hat{Y}, q)$ such that

$$
d W_{t}=\left(\gamma W_{t}-\delta\left(\left(1-q_{t}\right) B_{t}(\hat{Y}, q)-q_{t} W_{t}\right)\right) d t-d I_{t}+\beta_{t}(\hat{Y}, q)\left(d \hat{Y}_{t}-\left(\mu_{0}+\mu_{1} q_{t}\right) d t\right)+\left(\left(1-q_{t}\right) B_{t}(\hat{Y}, q)-q_{t} W_{t}\right) d N_{t} .
$$

This sensitivity $\beta_{t}(\hat{Y}, q)$ and the bonus process $B_{t}(\hat{Y}, q)$ are determined by the agent's past reports $\hat{Y}_{s}$, and riskiness of the project $q_{s}, 0 \leq s \leq t$.

The main difference from Lemma B is the jump $B_{t}$ in the agents continuation payoff that happens when the disaster occurs and the risk is low. Unlike the jump when the disaster outcome occurs, the size of bonus jump $B_{t}$ is an endogenous variable. We could add an additional randomization term in (30) as we did in Lemma B. However, as we argue later, randomization would not improve the contract.

As before, the agent has no incentives to steal cash flows if he gets at least $\lambda$ of promised value for each reported dollar, that is, if $\beta_{t} \geq \lambda$. This condition is not affected by the bonuses, which are conditional on the disaster process. However, the bonuses have a direct effect on the agent's incentives to gamble. In the high-risk regime, a disaster outcome results not only in the loss of the continuation payoff $W_{t}$ for the agent, but also in the loss of the bonus $B_{t}$ that the agent would get in the low-risk regime. On the other hand, the agent's benefits from switching to the high-risk regime are unaffected by the bonuses and are equal to an increase in the agent's continuation payoff by $\beta_{t} \rho d t$ over the period between time $t$ and $t+d t$. Thus, choosing the high-risk regime is incentive compatible if and only if $\beta_{t} \rho d t>\delta\left(W_{t}+B_{t}\right) d t$, or $W_{t}+B_{t}<\beta_{t} \frac{\rho}{\delta}$. The following lemma summarizes our conclusions.

LEMMA E: If the agent cannot save, truth-telling is incentive compatible if and only if $\beta_{t} \geq \lambda$ for all $t<\tau$. Moreover, if $\beta_{t} \geq \lambda$, it is optimal for the agent to choose the low-risk regime whenever $W_{t}+B_{t} \geq \beta_{t} \frac{\rho}{\delta}$ and switch to the high-risk regime whenever $W_{t}+B_{t}<\beta_{t} \frac{\rho}{\delta}$.

Proof of Lemma E: See Appendix. 
Lemma E states that positive bonuses conditional on the no-disaster outcome reduce the agent's incentive to choose the inefficient high-risk project. The minimum bonus needed to implement the low-risk regime is equal to $\min \left\{\beta_{t} \frac{\rho}{\delta}-W_{t}, 0\right\}$. Since the principal's expected payoff is a concave function of $W_{t}$, it is optimal to choose the smallest possible $\beta$ and $B$ that satisfy incentive compatibility and implement a desired level of risk. Thus, in the optimal contract, it should always be the case that $\beta_{t}=\lambda$. In addition, the optimal bonus should be $B_{t}=\min \left\{W^{S}-W_{t}, 0\right\}$ when the low-risk regime is implemented.

PROPOSITION 6: The contract that maximizes the principal's profit and delivers the value $W_{0} \in\left[0, W^{\mathrm{C}}\right]$ to the agent is characterized by three regions:

a. High-risk regime $\left(q_{t}=1\right)$ without bonuses when $W_{t} \in\left[0, W^{\mathrm{B}}\right)$

b. Low-risk regime $\left(q_{t}=0\right)$ with the bonus equal to $\left(W^{S}-W_{t}\right)$ when $W_{t} \in\left[W^{B}, W^{S}\right)$

c. Low-risk regime $\left(q_{t}=0\right)$ with no bonuses when $W_{t} \in\left[W^{S}, W^{C}\right]$

The agent's continuation payoff $W_{t}$ evolves according to

$$
d W_{t}=\left\{\begin{array}{lr}
(\gamma+\delta) W_{t} d t+\lambda\left(d \hat{Y}_{t}-(\mu+\rho) d t\right)-q_{t} W_{t} d N_{t} & \text { for } W_{t} \in\left[0, W^{B}\right) \\
\left((\gamma+\delta) W_{t}-\delta W^{S}\right) d t+\lambda\left(d \hat{Y}_{t}-\mu d t\right)+\left(\left(1-q_{t}\right)\left(W^{S}-W_{t}\right)-q_{t} W_{t}\right) d N_{t} & \text { for } W_{t} \in\left[W^{B}, W^{S}\right) \\
\gamma W_{t} d t-d I_{t}+\lambda\left(d \hat{Y}_{t}-\mu d t\right)-q_{t} W_{t} d N_{t} & \text { for } W_{t} \in\left[W^{S}, W^{C}\right]
\end{array}\right.
$$

When $W_{t} \in\left[0, W^{\mathrm{C}}\right), d I_{t}=0$. When $W_{t}=W^{\mathrm{C}}$, payments $d I_{t}$ cause $W_{t}$ to reflect at $W^{\mathrm{C}}$. If $W_{0}>W^{\mathrm{C}}$, an immediate payment $W_{0}-W^{\mathrm{C}}$ is made. The contract is terminated at time $\tau$, when $W_{t}$ reaches zero or the disaster outcome occurs. The principal's expected payoff at any point is given by a concave smooth function $p(W)$, which satisfies

$$
\begin{array}{ll}
(r+\delta) p(W)=(\mu+\rho-\delta(D-L))+(\gamma+\delta) W p^{\prime}(W)+\frac{1}{2} \lambda^{2} \sigma^{2} p^{\prime \prime}(W) & \text { for } W \in\left[0, W^{B}\right) \\
(r+\delta) p(W)=\mu+\delta p\left(W^{S}\right)+\left((\gamma+\delta) W-\delta W^{S}\right) p^{\prime}(W)+\frac{1}{2} \lambda^{2} \sigma^{2} p^{\prime \prime}(W) & \text { for } W \in\left[W^{B}, W^{S}\right) \\
r p(W)=\mu+\gamma W p^{\prime}(W)+\frac{1}{2} \lambda^{2} \sigma^{2} p^{\prime \prime}(W) & \text { for } W \in\left[W^{S}, W^{C}\right]
\end{array}
$$

The principal's continuation function $p(W)$ also satisfies the following boundary conditions $p(0)=L$ and $p^{\prime}\left(W^{C}\right)=-1$. The consumption boundary is determined by $r p\left(W^{C}\right)=\mu-\gamma W^{C}$, while the bonus region is determined by $W^{S}=\lambda \frac{\rho}{\delta}$ and $W^{B}$ that solve 


$$
L+\frac{\rho}{\delta}+W^{S} p^{\prime}\left(W^{B}\right)=D+p\left(W^{S}\right)
$$

Equation (80), which determines boundary $W^{B}$ of the gambling region, says that the second derivatives of the solutions of (77) and (78) match at $W^{B}$.

\section{Hidden Effort}

Throughout our analysis so far, we concentrated on the setting in which the cash flows are privately observed and the agent may divert them for his own consumption. In this section, we consider a principal-agent model in which the agent makes a hidden effort choice.

We now assume that the principal observes the cash flows, but not the agent's effort. A contract conditional on cash flow realizations determines the agent's compensation and termination of the project. Thus, there are two key changes to our model. First, since cash flows are observed, misreporting is not an issue. Second, we assume that at each point in time, the agent can choose his effort. Depending on agent's effort $\mu_{t}$, the resulting cash flow process is

$$
d Y_{t}=\left(\mu_{t}+\rho q_{t}\right) d t+\sigma d Z_{t}-D q_{t} d N_{t} .
$$

Working is costly for the agent, and the cost of effort is given by convex function $c(\mu)$. Specifically, we suppose that the agent's flow of utility is given by

$$
d C_{t}=d I_{t}-c\left(\mu_{t}\right) d t .
$$

The agent's choices of effort $\mu_{t}$ and risk $q_{t}$ are measurable processes with respect to past cash flow realizations. As in Section 3, we can define the agent's continuation utility after a history of realized cash flows $\left(Y_{s}, 0 \leq s \leq t\right)$ for given measurable process $\mu_{t}$ and $q_{t}$ of the agent's effort and risk choices:

$$
W_{t}=E_{t}\left[\int_{t}^{\tau} e^{-\gamma(s-t)}\left(d I_{s}-c\left(\mu_{t}\right) d t\right)\right] .
$$

It is straightforward to modify Lemmas $\mathrm{B}$ and $\mathrm{C}$ for the case with hidden effort.

LEMMA F: At any moment of time $t \leq \tau$, there is a sensitivity $\beta_{t}$ of the agent's continuation value towards cash flows realization such that

$$
d W_{t}=\left(\gamma+\delta q_{t}\right) W_{t} d t+c\left(\mu_{t}\right) d t-d I_{t}+\beta_{t}(Y)\left(d Y_{t}-\left(\mu_{t}+\rho q_{t}\right) d t\right)-q_{t} W_{t} d N_{t}
$$


Process $\beta$ is measurable with respect to the cash flow process $Y$. The agent's optimal choice of effort is given by $\mu_{t}=\arg \max _{\tilde{\mu}_{t}}\left\{\beta_{t} \tilde{\mu}_{t}-c\left(\tilde{\mu}_{t}\right)\right\}$. The agent chooses the low-risk regime whenever $W_{t} \geq \beta_{t} \frac{\rho}{\delta}$ and switch to the high-risk regime whenever $W_{t}<\beta_{t} \frac{\rho}{\delta}$.

Proof of Lemma F: See Appendix.

Given a contract in place, characterized by sensitivity $\beta_{t}(Y)$ of the agent's payoff toward the project's cash flows, the agent optimally chooses his effort and the riskiness of the project. For simplicity, we assume that the effort cost function $c$ is such that there is an internal solution for the optimal effort for any $\beta_{t}>0$. Thus, the optimal effort level for the agent is such that $c^{\prime}\left(\mu_{t}\right)=\beta_{t}$.

PROPOSITION 7: The contract that maximizes the principal's profit and delivers the value $W_{0} \in\left[0, W^{\mathrm{C}}\right]$ to the agent is characterized by two regions:

a. High-risk regime $\left(q_{t}=1\right)$ when $W_{t} \in\left[0, W^{\mathrm{G}}\right)$,

b. Low-risk regime $\left(q_{t}=0\right)$ when $W_{t} \in\left[W^{G}, W^{C}\right]$.

When $W_{t} \in\left[0, W^{\mathrm{C}}\right), d I_{t}=0$. When $W_{t}=W^{\mathrm{C}}$, payments $d I_{t}$ cause $W_{t}$ to reflect at $W^{\mathrm{C}}$. If $W_{0}>W^{\mathrm{C}}$, an immediate payment $W_{0}-W^{\mathrm{C}}$ is made. The contract is terminated at time $\tau$, when $W_{t}$ reaches zero or the disaster outcome occurs. The principal's expected payoff at any point is given by a concave smooth function $p(W)$, which satisfies

$$
\begin{array}{lr}
(r+\delta) p(W)=\mu(W)+\rho-\delta(D-L)+((\gamma+\delta) W+c(\mu(W))) p^{\prime}(W)+\frac{1}{2} c^{\prime}(\mu(W))^{2} \sigma^{2} p^{\prime \prime}(W) & \text { for } W \in\left[0, W^{G}\right) \\
r p(W)=\mu(W)+(\gamma W+c(\mu(W))) p^{\prime}(W)+\frac{1}{2} c^{\prime}(\mu(W))^{2} \sigma^{2} p^{\prime \prime}(W) & \text { for } W \in\left[W^{G}, W^{C}\right]
\end{array}
$$

The agent's optimal equilibrium effort $\mu(W)$ is given by

$$
\begin{aligned}
& \bar{\mu}(W) \text { s.t. } \quad \quad c^{\prime}(\bar{\mu}(W))=\frac{\delta W}{\rho} \quad \text { for } W \in\left[W^{G}, W^{H}\right), \\
& \mu^{*}(W)=\arg \max _{\tilde{\mu}} \tilde{\mu}+c(\tilde{\mu}) p^{\prime}(W)+\frac{1}{2} c^{\prime}(\tilde{\mu})^{2} \sigma^{2} p^{\prime \prime}(W) \text { for } W \in\left[0, W^{S}\right) \cup\left[W^{H}, W^{C}\right],
\end{aligned}
$$

where $W^{H}$ is such that

$$
\bar{\mu}\left(W^{H}\right)=\mu^{*}\left(W^{H}\right)
$$

and $W^{G}$ is such that function $p$ is twice continuously differentiable at $W^{G}$. 
The agent's continuation payoff $W_{t}$ evolves according to

$$
d W_{t}=\left(\left(\gamma+\delta q_{t}\right) W_{t}+c\left(\mu_{t}\right)\right) d t-d I_{t}+c^{\prime}\left(\mu_{t}\right)\left(d Y_{t}-\left(\mu_{t}+\rho q_{t}\right) d t\right)-q_{t} W_{t} d N_{t} .
$$

The principal's continuation function $p(W)$ also satisfies the following boundary conditions: $p(0)=L$ and $p^{\prime}\left(W^{C}\right)=-1$. The consumption boundary is determined by $r p\left(W^{C}\right)=\mu-\gamma W^{C}$.

\section{Conclusion}

This paper studies optimal incentive contracts in a setting with a two-dimensional moral hazard problem, in which an agent with limited liability privately chooses the riskiness of the project and can privately divert cash flows for consumption. Relative to the low risk project, the high risk project increases the probability of a high cash flow realization, but it also results in high losses in a bad state of nature, named "disaster".

To avoid risk-taking and diverting funds, investors must cede additional rents to the manager. In the static setting, we find that the optimal contract that implements the low-risk regime and truthful reporting of cash flows may require a very high level of compensation for the manager if the contract terms are contingent only on the reported cash flows. The expected level of the managerial compensation can be much lower if disaster states can be identified ex-post by paying the manager a large bonus if the firm survives. It can be implemented by giving the agent out-of-money put options on the companies that are likely to be ruined in the disaster state.

In a dynamic context, because managerial rents must be reduced following poor performance to prevent fund diversion, poorly performing managers will take on disaster risk even under an optimal contract. Even when disaster states can be identified ex-post, if performance is sufficiently weak the manager will forfeit eligibility for a bonus, and again take on disaster risk.

Our model can explain why suboptimal risk-taking can emerge even when investors are fully rational and managers are compensated optimally. Risk-taking is likely to take place when the disaster state is unlikely or after a history of poor performance, when the agent has little "skin" left in the game. As a result, the optimal contract has increased reliance on deferred 
compensation and provision of financial slack. Even absent evidence of risk-taking, small reductions in performance could lead to "harsh" punishments.

\section{APPENDIX}

\section{A Proofs}

\section{A.1 Proof of Lemma B}

Note that $\mathrm{W}_{\mathrm{t}}(\hat{Y}, q)$ is also the agent's promised value if $\hat{Y}_{\mathrm{s}}, 0 \leq \mathrm{s} \leq \mathrm{t}$, were the true cash flows and the agent reported truthfully. Therefore, without loss of generality we can prove (54) for the case in which the agent truthfully reports $\hat{Y}=Y$. In that case, by the law of iterated expectations

$$
V_{t}=\int_{0}^{t} e^{-\gamma s} d I_{s}(Y)+e^{-\gamma t} W_{t}(Y, q)
$$

is a martingale and by the martingale representation theorem there is a processes $\beta$ and $\psi$ such that

$$
d V_{t}=e^{-\gamma t} \beta_{t}(Y, q)\left(d Y_{t}-\left(\mu+\rho q_{t}\right) d t\right)+e^{-\gamma t} \psi_{t}(Y, q)\left(d N_{t}-\delta d t\right)+\left(d R_{t}(Y, q)-E\left[d R_{t}(Y, q)\right]\right),
$$

where $d Y_{t}-\left(\mu+\rho q_{t}\right) d t$ is a multiple of the standard Brownian motion for $t<\tau_{L} \wedge \tau_{D}$. If $d N_{t}=1$ and $q_{t}=1$, then $\psi_{t}=-W_{t}$, since the project liquidated immediately and the continuation payoff for the agent is zero. If $d N_{t}=1$ and $q_{t}=0$, then $\psi_{t}=0$, since the contractual payments to the agent cannot be conditional on the disaster process realizations that do not lead to the disaster outcome. Thus,

$$
\psi_{t}(Y, q)=-q_{t} W_{t}(Y, q) \text {. }
$$

Differentiating (A.1) with respect to $t$ we find

$$
\begin{aligned}
d V_{t}= & e^{-\gamma t} \beta_{t}(Y, q)\left(Y_{t}-\left(\mu+\rho q_{t}\right) d t\right)-e^{-\gamma t} q_{t} W_{t}(Y, q)\left(d N_{t}-\delta d t\right)+\left(d R_{t}(Y, q)-E\left[d R_{t}(Y, q)\right]\right)= \\
& e^{-\gamma t} d I_{t}(Y)-\gamma e^{-\gamma t} W_{t}(Y, q) d t+e^{-\gamma t} d W_{t}(Y, q),
\end{aligned}
$$

and thus (54) holds. $Q E D$

\section{A.2 Proof of Lemma C}

If the agent truthfully reports the cash flows and chooses the equilibrium risk level $q$, the present value of his future consumption is given by $V_{\tau}$. Then according (50) and (A.1) and Lemma B, his expected payoff is equal to 


$$
W_{0}=E\left[V_{\tau}\right]=E\left[V_{0}+\int_{0}^{\tau} e^{-\gamma t} \beta_{t}(Y, q)\left(d Y_{t}-\left(\mu+\rho q_{t}\right) d t\right)-\int_{0}^{\tau} e^{-\gamma t} q_{t} W_{t}(Y, q)\left(d N_{t}-\delta d t\right)+\int_{0}^{\tau}\left(d R_{t}-E\left[d R_{t}\right]\right)\right]
$$

Let $Y$ denote the cash flow corresponding to the equilibrium risk level $q$. If the agent reports $\hat{Y}_{t}$ and chooses risk level $\hat{q}_{t}$ at time $t$, he gains immediate income of $\lambda\left(d Y_{t}+\rho\left(\hat{q}_{t}-q_{t}\right) d t-d \hat{Y}\right)$, while the contract assumes risk level $q_{t}$ and treats the reported cash flow $\hat{Y}$ as the true cash flow. Since $E\left[d N_{t}\right]=\delta d t$, the agent's expected payoff under strategy $\hat{Y}$ and $\hat{q}$ is given by

$$
\begin{aligned}
& E\left[V_{0}+\int_{0}^{\tau} e^{-\gamma t} \beta_{t}(\hat{Y}, q)\left(d \hat{Y}_{t}-\left(\mu+\rho q_{t}\right) d t\right)+\lambda \int_{0}^{\tau} e^{-\gamma t}\left(d Y_{t}+\rho\left(\hat{q}_{t}-q_{t}\right) d t-d \hat{Y}_{t}\right)-\int_{0}^{\tau} e^{-\gamma t} W_{t}\left(\hat{q}_{t} d N_{t}-q_{t} \delta d t\right)\right]= \\
& E\left[V_{0}+\int_{0}^{\tau} e^{-\gamma t} \beta_{t}(\hat{Y}, q)\left(d Y_{t}+\rho\left(\hat{q}_{t}-q_{t}\right) d t-\left(\mu+\rho q_{t}\right) d t\right)+\int_{0}^{\tau} e^{-\gamma t}\left(\lambda-\beta_{t}(\hat{Y}, q)\right)\left(d Y_{t}+\rho\left(\hat{q}_{t}-q_{t}\right) d t-d \hat{Y}_{t}\right)\right]- \\
& E\left[\int_{0}^{\tau} e^{-\gamma t} \delta W_{t}\left(\hat{q}_{t}-q_{t}\right) d t\right]= \\
& W_{0}+E\left[\int_{0}^{\tau} e^{-\gamma t}\left(\lambda-\beta_{t}(\hat{Y}, q)\right)\left(d Y_{t}+\rho\left(\hat{q}_{t}-q_{t}\right) d t-d \hat{Y}_{t}\right)\right]+E\left[\int_{0}^{\tau} e^{-\gamma t}\left(\hat{q}_{t}-q_{t}\right)\left(\beta_{t}(\hat{Y}, q) \rho-\delta W_{t}\right) d t\right] .
\end{aligned}
$$

We can see from the first integral that if $\beta_{t} \geq \lambda$ for all $t$ then the payoff for the agent, who cannot overreport cash flows, is maximized when he truthfully reveals the cash flow, i.e., $d \hat{Y}_{t}=d Y_{t}+\rho\left(\hat{q}_{t}-q_{t}\right) d t$. If $\beta_{t}<\lambda$ on a set of positive measure, then the agent is better off underreporting on this set than always telling the truth.

From the second integral, we can see that the high risk is optimal for the agent when $\beta_{t}(\hat{Y}, q) \rho-\delta W>0$, or $W_{t}<\beta_{t}(\hat{Y}, q) \frac{\rho}{\delta}$, and the low risk is optimal when $W_{t} \geq \beta_{t}(\hat{Y}, q) \frac{\rho}{\delta} . Q E D$

\section{A.3 Proof of Proposition 5}

Let us prove that $p$ represents the principal's optimal profit, which is achieved by the contract outlined in the proposition. First, we note that $p$ is strictly concave on $\left[0, W^{P}\right)$ and $\left(W^{S}, W^{C}\right)$, and affine on $\left[W^{P}, W^{S}\right]$ and $\left[W^{C}, \infty\right)$

. We also note that because of the boundary conditions $p$ is twice differentiable.

Define

$$
G_{t} \equiv \int_{0}^{t} e^{-r s}\left(d Y_{s}-d I_{s}\right)+e^{-r t} p\left(W_{t}\right)
$$

According to Lemmas $\mathrm{B}$ and $\mathrm{C}$, under an arbitrary incentive compatible contract, $W_{t}$ evolves according to

$$
d W_{t}=\left(\gamma+\delta q_{t}\right) W_{t} d t-d I_{t}+\beta_{t} \sigma d Z_{t}-q_{t} W_{t} d N_{t}+\left(d R_{t}-E\left[d R_{t}\right]\right)
$$

Applying Ito's lemma gives 


$$
\begin{aligned}
e^{r t} d G_{t}= & \underbrace{\left(\mu+\rho q_{t}+\left(\gamma+\delta q_{t}\right) W_{t} p^{\prime}\left(W_{t}\right)+\frac{1}{2} \beta_{t}^{2} \sigma^{2} p^{\prime \prime}\left(W_{t}\right)-r p\left(W_{t}\right)+\delta q_{t}\left(L-D-p\left(W_{t}\right)\right)\right)}_{\leq 0} d t \\
& \underbrace{-\left(1+p^{\prime}\left(W_{t}\right)\right)}_{\leq 0} d I_{t}+\left(1+\beta_{t} p^{\prime}\left(W_{t}\right)\right) \sigma d Z_{t}+q_{t}\left(L-D-p\left(W_{t}\right)\right)\left(d N_{t}-\delta d t\right) \\
& +\underbrace{\left(p\left(W_{t}+J_{t}^{R}\right)-p\left(W_{t}\right)-p^{\prime}\left(W_{t}\right) J_{t}^{R}\right)}_{\leq 0} d \Delta_{t}^{R} .
\end{aligned}
$$

From (67)-(69) and the facts that $p\left(W_{t}\right)$ is weakly concave and $p^{\prime}\left(W_{t}\right) \geq-1, G_{t}$ is a supermartingale. It is a martingale if and only if $\beta_{t}=\lambda, W_{t} \leq W^{\mathrm{C}}$ for $t>0, I_{t}$ is increasing only when $W_{t} \geq W^{\mathrm{C}}$, and jumps of the size $J$ $=W^{S}-W^{P}$ can only happen when $W_{t}$ reaches thresholds $W^{P}$ or $W^{S}$. Indeed, since $p$ is strictly concave on $\left[W^{0}\right.$, $\left.W^{P}\right)$ and $\left[W^{S}, W^{C}\right)$, any jump outside of the interval $\left[W^{P}, W^{S}\right]$ makes the last term negative.

We can also show that jumps inside the interval $\left(W^{P}, W^{S}\right)$ make the first term in (A.7) negative. Indeed, because $p(W)$ and $W^{P}$ satisfy (67), (69) and (72), and $q=1$ for $W^{P}<W^{S}$, the drift simplifies to $(\gamma-r) p^{\prime}\left(W^{P}\right)\left(W_{t}-W^{P}\right) d t$ for $W_{t} \in\left(W^{P}, W^{S}\right)$, which is negative when $p^{\prime}\left(W^{P}\right)$ is negative. Differentiating (67) and taking into account the fact that $p^{\prime \prime}\left(W^{P}\right)=0$ gives $(\gamma-r) p^{\prime}\left(W^{P}\right)+0.5 \lambda^{2} \sigma^{2} p^{\prime \prime \prime}\left(W^{P}\right)=0$. Since $\mathrm{b}$ is strictly concave for $W<W^{P}, p^{\prime \prime \prime}\left(W^{P}\right)$ must be positive. Hence $p^{\prime}\left(W^{P}\right)$ is negative, and the drift is negative when $W_{t} \in\left(W^{P}, W^{S}\right)$. Thus, any jumps other than the ones between points $W^{P}$ and $W^{S}$ would make $G_{t}$ a supermartingale.

One can also verify using conditions (73) and (74) that the drift is negative when $W_{t}>W^{C}$.

We can now evaluate the principal's payoff for an arbitrary incentive compatible contract. Note that $p\left(W_{\tau}\right)=L-D 1_{\tau_{D} \leq \tau_{L}}$. For all $t<\infty$,

$$
\begin{aligned}
& E\left[\int_{0}^{\tau} e^{-r s}\left(d Y_{s}-d I_{s}\right)+e^{-r \tau}\left(L 1_{\tau_{L}<\tau_{D}}+(L-D) 1_{\tau_{D} \leq \tau_{L}}\right)\right] \\
& =E\left[G_{t \wedge \tau}+1_{t \leq \tau}\left(\int_{t}^{\tau} e^{-r s}\left(d Y_{s}-d I_{s}\right)+e^{-r \tau}\left(L 1_{\tau_{L}<\tau_{D}}+(L-D) 1_{\tau_{D} \leq \tau_{L}}\right)-e^{-r t} p\left(W_{t}\right)\right)\right] \\
& =\underbrace{E\left[G_{t \wedge \tau}\right]}_{\leq G_{0}=p\left(W_{0}\right)}+e^{-r t} E\left[1_{t \leq \tau}(\underbrace{E_{t}\left[\int_{t}^{\tau} e^{-r(s-t)}\left(d Y_{s}-d I_{s}\right)+e^{-r(\tau-t)}\left(L 1_{\tau_{L}<\tau_{D}}+(L-D) 1_{\tau_{D} \leq \tau_{L}}\right)\right]}_{\leq \mu / r-W_{t}=\text { first best }}-p\left(W_{t}\right)] .\right.
\end{aligned}
$$

Now, since $p^{\prime}(W) \geq-1, \mu / r-W-p(W) \leq \mu / r-L$. Therefore, letting $t \rightarrow \infty$,

$$
E\left[\int_{0}^{\tau} e^{-r s}\left(d Y_{s}-d I_{s}\right)+e^{-r \tau}\left(L 1_{\tau_{L}<\tau_{D}}+(L-D) 1_{\tau_{D} \leq \tau_{L}}\right)\right] \leq p\left(W_{0}\right)
$$


Thus, for a contract that satisfies the conditions of the proposition, $G_{t}$ is a martingale until time $\tau$ because $p^{\prime}\left(W_{t}\right)$ stays bounded. Therefore, the payoff $p\left(W_{0}\right)$ is achieved with equality.

Finally, we verify that conditions (70) and (71) lead to the corner solutions for $W^{P}$. If $W^{P} \in\left(0, W^{S}\right)$, it must solve (72), which is equivalent to the condition that $p^{\prime \prime}\left(W^{P}\right)=0$. Equation (72) can be rewritten as follows

$$
(r+\delta)\left(p\left(W^{P}\right)-p^{\prime}\left(W^{P}\right) W^{P}-L\right)-(\gamma-r) p^{\prime}\left(W^{P}\right) W^{P}=\mu+\rho-(\delta D+r L) .
$$

The left hand side is positive because $p$ is concave and $p^{\prime}\left(W^{P}\right)$ is negative and is equal to zero when $W^{P}=0$. Thus, when $\mu+\rho<\delta D+r L$, there is no internal solution and $W^{P}=0$. On the other hand, the solution of (67) is concave at $W^{S}$, i.e., $p^{\prime}\left(W^{S}\right) \leq 0$, only when (71) is satisfied. In this case, the value function is concave everywhere and public randomization cannot improve the contract. $Q E D$

\section{A.4 Proof of Lemma E}

If the agent truthfully reports the cash flows and chooses the equilibrium risk level $q$, then according to relations (50), (A.1) and Lemma D, his expected payoff is equal to

$$
\begin{aligned}
W_{0}=E\left[V_{\tau}\right]= & E\left[V_{0}+\int_{0}^{\tau} e^{-\gamma t} \beta_{t}(Y, q)\left(d Y_{t}-\left(\mu+\rho q_{t}\right) d t\right)+\int_{0}^{\tau} e^{-\gamma t}\left(\left(1-q_{t}\right) B_{t}(Y, q)-q_{t} W_{t}\right)\left(d N_{t}-\delta d t\right)\right]= \\
& E\left[V_{0}+\int_{0}^{\tau} e^{-\gamma t} \beta_{t}(Y, q)\left(d Y_{t}-\left(\mu+\rho q_{t}\right) d t\right)\right] .
\end{aligned}
$$

Let $Y$ denote the cash flow corresponding to the equilibrium risk level $q$. If the agent reports $\hat{Y}_{t}$ and chooses risk level $\hat{q}_{t}$ at time $t$, he gains immediate income of $\lambda\left(d Y_{t}+\rho\left(\hat{q}_{t}-q_{t}\right) d t-d \hat{Y}\right)$, while the contract assumes risk level $q_{t}$ and treats the reported cash flow $\hat{Y}$ as the true cash flow. Since $E\left[d N_{t}\right]=\delta d t$, the agent's expected payoff under reporting strategy $\hat{Y}$ is given by 


$$
\begin{aligned}
& E\left[V_{0}+\int_{0}^{\tau} e^{-\gamma t} \beta_{t}(\hat{Y}, q)\left(d \hat{Y}_{t}-\left(\mu+\rho q_{t}\right) d t\right)+\lambda \int_{0}^{\tau} e^{-\gamma t}\left(d Y_{t}+\rho\left(\hat{q}_{t}-q_{t}\right) d t-d \hat{Y}_{t}\right)\right]+ \\
& E\left[\int_{0}^{\tau} e^{-\gamma t}\left(\left(\left(1-\hat{q}_{t}\right) B_{t}(\hat{Y}, q)-\hat{q}_{t} W_{t}\right) d N_{t}-\left(\left(1-q_{t}\right) B_{t}(\hat{Y}, q)-q_{t} W_{t}\right) \delta d t\right)\right]= \\
& E\left[V_{0}+\int_{0}^{\tau} e^{-\gamma t} \beta_{t}(\hat{Y}, q)\left(d Y_{t}+\rho\left(\hat{q}_{t}-q_{t}\right) d t-\left(\mu+\rho q_{t}\right) d t\right)+\int_{0}^{\tau} e^{-\gamma t}\left(\lambda-\beta_{t}(\hat{Y}, q)\right)\left(d Y_{t}+\rho\left(\hat{q}_{t}-q_{t}\right) d t-d \hat{Y}_{t}\right)\right]- \\
& E\left[\int_{0}^{\tau} e^{-\gamma t}\left(\hat{q}_{t}-q_{t}\right)\left(W_{t}+B_{t}(\hat{Y}, q)\right) \delta d t\right]= \\
& W_{0}+E\left[\int_{0}^{\tau} e^{-\gamma t}\left(\lambda-\beta_{t}(\hat{Y}, q)\right)\left(d Y_{t}+\rho\left(\hat{q}_{t}-q_{t}\right) d t-d \hat{Y}_{t}\right)\right]+E\left[\int_{0}^{\tau} e^{-\gamma t}\left(\hat{q}_{t}-q_{t}\right)\left(\beta_{t}(\hat{Y}, q) \rho-\delta\left(W_{t}+B_{t}(\hat{Y}, q)\right)\right) d t\right] .
\end{aligned}
$$

We see from the first integral that if $\beta_{t} \geq \lambda$ for all $t$ then the agent's payoff is maximized when the agent chooses $d \hat{Y}_{t}=d Y_{t}+\mu_{1}\left(\hat{q}_{t}-q_{t}\right) d t$, since the agent cannot over-report cash flows. If $\beta_{t}<\lambda$ on a set of positive measure, then the agent is better off underreporting on this set than always telling the truth.

It directly follows from the second integral that the high risk is optimal for the agent either when $\beta_{t}(\hat{Y}, q) \rho-\delta\left(W_{t}+B_{t}(\hat{Y}, q)\right)>0$, or when $W_{t}+B_{t}(\hat{Y}, q)<\beta_{t}(\hat{Y}, q) \frac{\rho}{\delta}$, and that the low risk is optimal when $W_{t}+B_{t}(\hat{Y}, q) \geq \beta_{t}(\hat{Y}, q) \frac{\rho}{\delta} \cdot Q E D$

\section{A.5 Proof of Proposition 6}

Let us prove that $p$ represents the principal's optimal profit, which is achieved by the contract outlined in the proposition. First, we note that $p$ is concave on $\left[0, W^{C}\right]$. Indeed, when $W_{t}=W^{S}$ equation (78) becomes identical to (79), and condition (80) implies that the second derivatives of the solutions of (77) and (78) agree at $W^{B}$.

Define

$$
G_{t} \equiv \int_{0}^{t} e^{-r s}\left(d Y_{s}-d I_{s}\right)+e^{-r t} p\left(W_{t}\right)
$$

According to Lemma $\mathrm{D}$, under an arbitrary incentive compatible contract, $W_{t}$ evolves according to

$$
d W_{t}=\left(\gamma W_{t}+\delta q_{t} W_{t}-\delta\left(1-q_{t}\right) B_{t}\right) d t-d I_{t}+\beta_{t} \sigma d Z_{t}+\left(-q_{t} W_{t}+\left(1-q_{t}\right) B_{t}\right) d N_{t} .
$$

Applying Ito's lemma gives 


$$
\begin{aligned}
e^{r t} d G_{t}= & \underbrace{\left(\mu+\rho q_{t}+\left(\gamma+\delta q_{t}\right) W_{t} p^{\prime}\left(W_{t}\right)+\frac{1}{2} \beta_{t}^{2} \sigma^{2} p^{\prime \prime}\left(W_{t}\right)-r p\left(W_{t}\right)+\delta\left(q_{t}\left(L-D-p\left(W_{t}\right)\right)+\left(1-q_{t}\right)\left(p\left(W_{t}+B_{t}\right)-p\left(W_{t}\right)-B_{t} p^{\prime}\left(W_{t}\right)\right)\right)\right.}_{\leq 0} d t \\
& \underbrace{-\left(1+p^{\prime}\left(W_{t}\right)\right)}_{\leq 0} d I_{t}+\left(1+\beta_{t} p^{\prime}\left(W_{t}\right)\right) \sigma d Z_{t}+\left(q_{t}\left(L-D-p\left(W_{t}\right)\right)+\left(1-q_{t}\right)\left(p\left(W_{t}+B_{t}\right)-p\left(W_{t}\right)\right)\left(d N_{t}-\delta d t\right) .\right.
\end{aligned}
$$

According to Lemma E, $q_{t}=0$ if and only if $W_{t}+B_{t} \geq \beta_{t} \frac{\rho}{\delta}$. Since $p$ solves (77)-(79) and, the drift is zero and $G_{t}$ is a martingale when $\beta_{t}=\lambda, I_{t}$ is increasing only when $W_{t} \geq W^{\mathrm{C}}$, and $B_{t}=W^{S}-W_{t}$ for $W_{t} \in\left[W^{B}, W^{S}\right]$ and $B_{t}=0$ for $W_{t} \in\left[0, W^{B}\right] \cup\left[W^{S}, \infty\right]$. Since $p$ is concave the drift is negative when $\beta_{t}$ $>\lambda$, or $B_{t} \neq W^{S}-W_{t}$ for $W_{t} \in\left[W^{B}, W^{S}\right]$. The drift is also negative when $B_{t}=W^{S}-W_{t}$ for $W_{t}<W^{B}$. Indeed, since in this case $q_{t}=0, p$ solves (77) and $W^{B}$ solves (80), the drift is equal to $-\delta\left(L+\frac{\rho}{\delta}+W^{S} p^{\prime}\left(W_{t}\right)-D-p\left(W^{S}\right)\right) d t=-\delta W^{S}\left(p^{\prime}\left(W_{t}\right)-p^{\prime}\left(W^{B}\right)\right) d t<0$.

We can now evaluate the principal's payoff for an arbitrary incentive compatible contract. Note that $b\left(W_{\tau}\right)=L-M 1_{\tau_{D} \leq \tau_{L}}$. For all $t<\infty$,

$$
\begin{aligned}
& E\left[\int_{0}^{\tau} e^{-r s}\left(d Y_{s}-d I_{s}\right)+e^{-r \tau}\left(L 1_{\tau_{L}<\tau_{D}}+(L-D) 1_{\tau_{D} \leq \tau_{L}}\right)\right] \\
& =E\left[G_{t \wedge \tau}+1_{t \leq \tau}\left(\int_{t}^{\tau} e^{-r s}\left(d Y_{s}-d I_{s}\right)+e^{-r \tau}\left(L 1_{\tau_{L}<\tau_{D}}+(L-D) 1_{\tau_{D} \leq \tau_{L}}\right)-e^{-r t} p\left(W_{t}\right)\right)\right] \\
& =\underbrace{E\left[G_{t \wedge \tau}\right]}_{\leq G_{0}=p\left(W_{0}\right)}+e^{-r t} E\left[1_{t \leq \tau}(\underbrace{E_{t}\left[\int_{t}^{\tau} e^{-r(s-t)}\left(d Y_{s}-d I_{s}\right)+e^{-r(\tau-t)}\left(L 1_{\tau_{L}<\tau_{D}}+(L-D) 1_{\tau_{D} \leq \tau_{L}}\right)\right]}_{\leq \mu / r-W_{t}=\text { first best }}-p\left(W_{t}\right)] .\right.
\end{aligned}
$$

Now, since $p^{\prime}(W) \geq-1, \mu / r-W-p(W) \leq \mu / r-L$. Therefore, letting $t \rightarrow \infty$,

$$
E\left[\int_{0}^{\tau} e^{-r s}\left(d Y_{s}-d I_{s}\right)+e^{-r \tau}\left(L 1_{\tau_{L}<\tau_{D}}+(L-D) 1_{\tau_{D} \leq \tau_{L}}\right)\right] \leq p\left(W_{0}\right)
$$

Finally, for a contract that satisfies the conditions of the proposition, $G_{t}$ is a martingale until time $\tau$ because $p^{\prime}\left(W_{t}\right)$ stays bounded. Therefore, the payoff $p\left(W_{0}\right)$ is achieved with equality. $Q E D$

\section{A.6 Proof of Lemma F}

The process 


$$
V_{t}=\int_{0}^{t} e^{-\gamma s}\left(d I_{s}-c\left(\mu_{s}\right) d s\right)+e^{-\gamma t} W_{t}
$$

is a martingale and by the martingale representation theorem there is a processes $\beta$ such that

$$
d V_{t}=e^{-\gamma t} \beta_{t}\left(d Y_{t}-\left(\mu_{t}+\rho q_{t}\right) d t\right)-e^{-\gamma t} q_{t} W_{t}\left(d N_{t}-\delta d t\right),
$$

where $d Y_{t}-\left(\mu_{t}+\rho q_{t}\right) d t$ is a multiple of the standard Brownian motion for $t<\tau_{L} \wedge \tau_{D}$.

Differentiating (A.81) with respect to $t$ we find

$$
d V_{t}=e^{-\gamma t} d I_{t}-c\left(\mu_{t}\right) d t-\gamma e^{-\gamma t} W_{t} d t+e^{-\gamma t} d W_{t} .
$$

Comparing (A.83) to (A.82) gives

$$
d W_{t}=\left(\gamma+\delta q_{t}\right) W_{t} d t+c\left(\mu_{t}\right) d t-d I_{t}+\beta_{t}\left(d Y_{t}-\left(\mu_{t}+\rho q_{t}\right) d t\right)-q_{t} W_{t} d N_{t},
$$

and thus (90) holds.

If the agent chooses the equilibrium risk $q$ and effort $\mu$ levels (as specified in Lemma F), then according to (A.82), his expected payoff is equal to

$$
W_{0}=E\left[V_{\tau}\right]=E\left[V_{0}+\int_{0}^{\tau} e^{-\gamma t} \beta_{t}\left(d Y_{t}-\left(\mu+\rho q_{t}\right) d t\right)-\int_{0}^{\tau} e^{-\gamma t} q_{t} W_{t}\left(d N_{t}-\delta d t\right)\right]=E\left[V_{0}\right]
$$

Let $Y$ denote the cash flow corresponding to the equilibrium risk $q$ and effort $\mu$ levels. If the agent chooses risk $\hat{q}_{t}$ and effort $\hat{\mu}_{t}$ at time $t$, the cash flow realization will be $d \hat{Y}_{t}=d Y_{t}+\left(\hat{\mu}_{t}-\mu_{t}\right) d t+\rho\left(\hat{q}_{t}-q_{t}\right) d t$ and the cost of effort will be reduced by $\left(c\left(\mu_{t}\right)-c\left(\hat{\mu}_{t}\right)\right) d t$. Hence, the agent's expected payoff would be

$$
\begin{aligned}
& E\left[V_{0}+\int_{0}^{\tau} e^{-\gamma t} \beta_{t}(\hat{Y})\left(d Y_{t}+\left(\hat{\mu}_{t}-\mu_{t}\right) d t+\mu_{1}\left(\hat{q}_{t}-q_{t}\right) d t-\left(\mu_{t}+\rho q_{t}\right) d t\right)+\int_{0}^{\tau} e^{-\gamma t}\left(c\left(\mu_{t}\right)-c\left(\hat{\mu}_{t}\right)\right) d t\right]- \\
& E\left[\int_{0}^{\tau} e^{-\gamma t} W_{t}\left(\hat{q}_{t} d N_{t}-q_{t} \delta d t\right)\right]= \\
& E\left[V_{0}+\int_{0}^{\tau} e^{-\gamma t} \beta_{t}(\hat{Y})\left(d Y_{t}-\mu_{t} d t-\rho q_{t} d t\right)\right]+E\left[\int_{0}^{\tau} e^{-\gamma t}\left(\left(\beta_{t}(\hat{Y}) \hat{\mu}_{t}-c\left(\hat{\mu}_{t}\right)\right)-\left(\beta_{t}(\hat{Y}) \mu_{t}-c\left(\mu_{t}\right)\right)\right) d t\right]+ \\
& E\left[\int_{0}^{\tau} e^{-\gamma t}\left(\hat{q}_{t}-q_{t}\right)\left(\beta_{t}(\hat{Y}) \rho-\delta W_{t}\right) d t\right] \leq E\left[V_{0}\right]
\end{aligned}
$$

The first equality follows from the fact that process $N$ is independent of $Y$ and $E\left[d N_{t}\right]=\delta d t$. The expected payoff for the agent is less than or equal to $E\left[V_{0}\right]$ because the first integral is a martingale; the second integral is non-positive since $\mu_{t}=\arg \max _{\tilde{\mu}_{t}}\left\{\beta_{t} \tilde{\mu}_{t}-c\left(\tilde{\mu}_{t}\right)\right\}$; and the last integral cannot be positive because $\hat{q}_{t} \in\{0,1\}$ and $q_{t}=1$ if and only if $\beta_{t}(\hat{Y}) \rho-\delta W_{t}>0$. Thus, the expected payoff for the agent reaches $E\left[V_{0}\right]$ only when $\hat{\mu}_{t}=\mu_{t}=\arg \max _{\tilde{\mu}_{t}}\left\{\beta_{t} \tilde{\mu}_{t}-c\left(\tilde{\mu}_{t}\right)\right\}$ and $\hat{q}_{t}=q_{t}=1_{\left\{\beta_{t}(\hat{Y}) \rho-\delta W_{t}>0\right\}} \cdot Q E D$

\section{A.7 Proof of Proposition 7}


Let us prove that $p$ represents the principal's optimal profit, which is achieved by the contract outlined in the proposition. First, we note that $p$ is concave on $\left[0, W^{C}\right)$. We also note that because of the boundary conditions $p$ is twice differentiable.

Define

$$
G_{t} \equiv \int_{0}^{t} e^{-r s}\left(d Y_{s}-d I_{s}\right)+e^{-r t} p\left(W_{t}\right)
$$

According to Lemma $\mathrm{F}$, under an arbitrary incentive compatible contract, $W_{t}$ evolves according to

$$
d W_{t}=\left(\gamma+\delta q_{t}\right) W_{t} d t+c\left(\mu_{t}\right) d t-d I_{t}+c^{\prime}\left(\mu_{t}\right) \sigma d Z_{t}-q_{t} W_{t} d N_{t}
$$

Applying Ito's lemma gives

$$
\begin{aligned}
e^{r t} d G_{t}= & \left(A\left(q_{t}, \mu_{t}, W_{t}\right)+\frac{1}{2}\left(c^{\prime}\left(\mu_{t}\right)\right)^{2} \sigma^{2} p^{\prime \prime}\left(W_{t}\right)\right) d t \\
& -\left(1+p^{\prime}\left(W_{t}\right)\right) d I_{t}+\left(1+c^{\prime}\left(\mu_{t}\right) p^{\prime}\left(W_{t}\right)\right) \sigma d Z_{t}+q_{t}\left(L-D-p\left(W_{t}\right)\right)\left(d N_{t}-\delta d t\right),
\end{aligned}
$$

where

$$
A\left(q_{t}, \mu_{t}, W_{t}\right)=\mu_{t}+\rho q_{t}+\left(\left(\gamma+\delta q_{t}\right) W_{t}+c\left(\mu_{t}\right)\right) p^{\prime}\left(W_{t}\right)-r p\left(W_{t}\right)+\delta q_{t}\left(L-D-p\left(W_{t}\right)\right) .
$$

From (91)-(95) and the facts that $p\left(W_{t}\right)$ is concave and $p^{\prime}\left(W_{t}\right) \geq-1, G_{t}$ is a supermartingale, and it is a martingale if and only if $\mu_{t}$ is given by (93) and (94), $W_{t} \leq W^{\mathrm{C}}$ for $t>0$, and $I_{t}$ is increasing only when $W_{t} \geq W^{\mathrm{C}}$.

We verify that the optimality condition for $W^{G}$ requires that the second derivative of $p$ remains continuous at $W^{G}$. In order for $G_{t}$ to be a supermartingale, it has to be the case that

$0=A\left(1, \mu^{*}, W\right)+\frac{1}{2}\left(c^{\prime}\left(\mu^{*}\right)\right)^{2} \sigma^{2} p^{\prime \prime}(W) \geq A(0, \bar{\mu}, W)+\frac{1}{2}\left(c^{\prime}(\bar{\mu})\right)^{2} \sigma^{2} p^{\prime \prime}(W)$ for $W<W^{G}$,

and

$$
A\left(1, \mu^{*}, W\right)+\frac{1}{2}\left(c^{\prime}\left(\mu^{*}\right)\right)^{2} \sigma^{2} p^{\prime \prime}(W) \leq A(0, \bar{\mu}, W)+\frac{1}{2}\left(c^{\prime}(\bar{\mu})\right)^{2} \sigma^{2} p^{\prime \prime}(W)=0, \text { for } W>W^{G} .
$$

Thus, $p^{\prime \prime}(W) \leq-\frac{A(0, \bar{\mu}(W), W)-A\left(1, \mu^{*}(W), W\right)}{\frac{1}{2} \sigma^{2}\left(\left(c^{\prime}(\bar{\mu}(W))\right)^{2}-\left(c^{\prime}\left(\mu^{*}(W)\right)\right)^{2}\right)}$ for $W<W^{G}$,

and

$$
p^{\prime \prime}(W) \geq-\frac{A(0, \bar{\mu}(W), W)-A\left(1, \mu^{*}(W), W\right)}{\frac{1}{2} \sigma^{2}\left(\left(c^{\prime}(\bar{\mu}(W))\right)^{2}-\left(c^{\prime}\left(\mu^{*}(W)\right)\right)^{2}\right)} \text { for } W>W^{G} \text {. }
$$


Since $\bar{\mu}(W), \mu^{*}(W)$, and $A(q, \mu, W)$ are continuous functions of $W, p$ must have a continuous second derivative at $W^{G}$.

Note that $p\left(W_{\tau}\right)=L-D 1_{\tau_{D} \leq \tau_{L}}$. Thus, the principal's payoff for an arbitrary incentive compatible contract

$$
E\left[\int_{0}^{\tau} e^{-r s}\left(d Y_{s}-d I_{s}\right)+e^{-r \tau}\left(L 1_{\tau_{L}<\tau_{D}}+(L-D) 1_{\tau_{D} \leq \tau_{L}}\right)\right]=E\left[G_{\tau}\right] \leq E\left[G_{0}\right]=p\left(W_{0}\right) .
$$

The inequality follows from the fact that $G$ is a supermartingale for an arbitrary incentive compatible contract. For a contract that satisfies the conditions of the proposition, $G_{t}$ is a martingale until time $\tau$. Therefore, the payoff $p\left(W_{0}\right)$ is achieved with equality. $Q E D$ 


\section{REFERENCES}

Basak, S., Pavlova, A., and A. Shapiro, 2007, Optimal Asset Allocation and Risk Shifting in Money Management, Review of Financial Studies 20, 1583-1621.

Biais. B. and C. Casamatta, 1999, Optimal Leverage and Aggregate Investment, Journal of Finance 54, Papers and Proceedings, Fifty-Ninth Annual Meeting, American Finance Association, New York, New York, January 4-6 1999, 1291-1323.

Cadenillas, A., Cvitanic, J., and F. Zapatero, 2007, Optimal risk-sharing with effort and project choice, Journal of Economic Theory 133, 403-440.

Carpenter, J.N., 2000, Does Option Compensation Increase Managerial Risk Appetite? Journal of Finance 55, 2311-2331.

DeMarzo, P. and M. Fishman, 2007a, Optimal Long-Term Financial Contracting, Review of Financial Studies 20, 2079-2128.

DeMarzo, P. and M. Fishman, 2007b, Agency and Optimal Investment Dynamics, Review of Financial Studies 20, 151-188.

Diamond, P., 1998, Managerial Incentives: On the Near Linearity of Optimal Compensation, Journal of Political Economy 106, 931-957.

Hellwig, M., 2009, A Reconsideration of the Jensen-Meckling Model of Outside Finance, Journal of Financial Intermediation 18, 495-525.

Makarov, I. and G. Plantin, 2010, Rewarding Trading Skills Without Inducing Gambling, Working Paper, London Business School.

Meckling, W., and M. Jensen, 1976, Theory of the Firm: Managerial Behavior, Agency Costs and Ownership Structure, Journal of Financial Economics 3, 305-360.

Ou-Yang, H., 2003, Optimal Contracts in a Continuous-Time Delegated Portfolio Management Problem, Review of Financial Studies 16, 173-208.

Palomino, F. and A. Prat, 2003, Risk Taking and Optimal Contracts for Money Managers, Rand Journal of Economics 34, 113-137.

Ross, S., 2004, Compensation, Incentives, and the Duality of Risk Aversion and Riskiness, Journal of Finance 59, 207-225.

Salanie P., 1997, The Economics of Contracts: A Primer. MIT Press, Cambridge, Mass. 